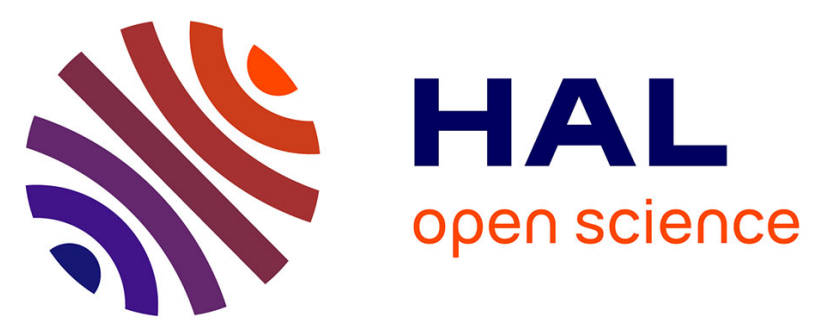

\title{
Influence of balloon design, plaque material composition, and balloon sizing on acute post angioplasty outcomes: An implicit finite element analysis
}

Bernard Helou, Aline Bel-Brunon, Claire Dupont, Wenfeng Ye, Claudio Silvestro, Michel Rochette, Antoine Lucas, Adrien Kaladji, Pascal Haigron

\section{To cite this version:}

Bernard Helou, Aline Bel-Brunon, Claire Dupont, Wenfeng Ye, Claudio Silvestro, et al.. Influence of balloon design, plaque material composition, and balloon sizing on acute post angioplasty outcomes: An implicit finite element analysis. International Journal for Numerical Methods in Biomedical Engineering, 2021, 37 (8), pp.e03499. 10.1002/cnm.3499 . hal-03268327

\section{HAL Id: hal-03268327 https://hal.science/hal-03268327}

Submitted on 1 Jul 2021

HAL is a multi-disciplinary open access archive for the deposit and dissemination of scientific research documents, whether they are published or not. The documents may come from teaching and research institutions in France or abroad, or from public or private research centers.
L'archive ouverte pluridisciplinaire $\mathbf{H A L}$, est destinée au dépôt et à la diffusion de documents scientifiques de niveau recherche, publiés ou non, émanant des établissements d'enseignement et de recherche français ou étrangers, des laboratoires publics ou privés. 


\section{Title}

Influence of balloon design, plaque material composition, and balloon sizing on acute post angioplasty outcomes: an implicit Finite Element Analysis

Authors and affiliations:

Bernard Helou ${ }^{1}$, Aline Bel-Brunon ${ }^{2}$, Claire Dupont ${ }^{1}$, Wenfeng $\mathrm{Ye}^{3}$, Claudio Silvestro ${ }^{4}$, Michel Rochette ${ }^{3}$, Antoine Lucas ${ }^{1}$, Adrien Kaladji ${ }^{1}$, and Pascal Haigron ${ }^{1}$

${ }^{1}$ Univ Rennes, CHU Rennes, Inserm, LTSI - UMR 1099, F-35000 Rennes, France; ${ }^{2}$ Univ Lyon, INSA-Lyon, CNRS UMR5259, LaMCoS, F-69621, France; ${ }^{3}$ ANSYS, Villeurbanne F69100, France; ${ }^{4}$ Medtronic, Aortic Peripheral \& Venous (APV) Group, Santa Rosa, CA 95403, USA

\section{Abstract and key terms}

In this work we propose a generic modeling approach for simulating percutaneous transluminal angioplasty (PTA) endovascular treatment, and evaluating the influence of balloon design, plaque composition, and balloon sizing on acute post-procedural outcomes right after PTA, without stent implantation. Clinically-used PTA balloons were classified into two categories according to their compliance characteristics, and were modeled correspondingly. Self-defined elastoplastic constitutive laws were implemented within the plaque and artery models, after calibration based on experimental and clinical data. Finite element method (FEM) implicit solver was used to simulate balloon inflation and deflation. Besides balloon profile at max inflation, results are mainly assessed in terms of the elastic recoil ratio (ERR) and lumen gain ratio (LGR) obtained immediately after PTA. No variations in ERR nor LGR values were detected when the balloon design changed, despite the differences observed in their profile at max inflation. Moreover, LGR and ERR inversely varied with the augmentation of calcification level within the plaque $(-11 \%$ vs. $+4 \%$ respectively, from fully lipidic to fully calcified plaque). Furthermore, results showed a direct correlation between balloon sizing and LGR and ERR, with noticeably higher rates of change for LGR $(+18 \%$ and $+2 \%$ for LGR and ERR respectively for a calcified plaque and a balloon pressure increasing from 10 to $14 \mathrm{~atm})$. However a larger LGR comes with a higher risk of arterial rupture. This proposed methodology opens the way for evaluation of angioplasty balloon selections towards clinical procedure optimization.

Keywords: Atherosclerosis, Balloon angioplasty, Balloon design, Plaque composition, Balloon sizing, Acute post-procedural outcomes, Finite element analysis.

This article has been accepted for publication and undergone full peer review but has not been through the copyediting, typesetting, pagination and proofreading process which may lead to differences between this version and the Version of Record. Please cite this article as doi: 10.1002/cnm.3499 


\section{Introduction}

Atherosclerosis is one of the most common vascular pathologies in the world, with high mortality rates. This pathogenesis is complex in general. In a simplified description, it could be defined as the accumulation first of lipids (mainly cholesterol) at the arterial inner wall, followed by the growth of the plaque with time forming a stiffer structure composed of connective tissues, collagen fibers, and calcifications [1]. The plaque partly or totally occludes the lumen, affecting normal blood circulation, and can lead to severe consequences on perfused organs.

Several surgical and endovascular treatment techniques are clinically possible for atherosclerosis, including percutaneous transluminal angioplasty (PTA), endarterectomy and arterial bypass. PTA, which is the minimally-invasive option, is a favored alternative to invasive treatments in order to avoid complications experienced with conventional surgeries [2], specifically for patients at higher risks of surgical morbidity and mortality [3]. Treatment via PTA consists of inserting percutaneously a balloon catheter within the diseased vessel region, followed by inflating the balloon in order to compress/damage the plaque and enlarge the vessel lumen [4-6]. Stenting might be subsequently required for some treated cases. Their usage generally depends on the lumen patency obtained immediately after PTA [7]. Stent implantation has won increased significance over the past years by showing better clinical outcomes in terms of preventing elastic recoil compared to PTA alone [8]. However, being associated to high rates of vessel injuries, thrombogenicity in addition to in-stent restenosis (e.g. $20-40 \%$ of patients at 2 years in femoral arteries [3]), stents implantation after PTA is being re-questioned. Furthermore, small arterial dimensions and complex kinematics [9] (flexions and repetitive stresses especially in peripheral arteries [10]) favor the mechanical fracture of stents, calling for specific decisions for their usage. Two main reasons explain the focus of this work on PTA outcomes without stent implantation: 1) For certain arteries within the arterial tree such as the femoropopliteal for example, a policy of conventional plain balloon angioplasty appears to be the reasonable initial treatment, with other adjunctive techniques (such as stenting and local drug delivery) reserved to when angioplasty results are suboptimal or when restenosis occurs [11]. This means that, even though it is still not explicitly stated within international publications covering consensus for peripheral arterial disease management (i.e. TASC II), in practical terms, PTA is usually the initial revascularization approach for atherosclerosis in most lower extremity arteries [12]. Moreover, it continues to be the technique all other endovascular treatment modalities are compared against [13]. 2) Due to recent medical device innovations including catheters with an "active" mode of action, drug-coated balloons (DCBs) are being lately introduced as minimally-invasive endovascular treatments for stenosed arteries as well. DCBs combine standard balloon angioplasty and anti-proliferative drug technology. These devices use drug substances such as paclitaxel that would be released towards the vessel wall during deployment. With their possible advantage in reducing restenosis rates, not leaving foreign object behind in the body (e.g. stent), and their ability to be used in anatomy where stent implantation might not be feasible (e.g., across the knee joint) [13], the interest in studying the balloon angioplasty treatment with the lumen gains and elastic recoils associated to it is back on track, as is the case in our hospital center. 
The success of PTA at short term is assessed by the residual lumen gained immediately after the endovascular treatment. Besides the compression/damage within the plaques, nonrecoverable deformation also occurs in the arteries (clinically termed "controlled vessel injury" [14]). The resulting deformation is usually evaluated by either the patency rate or the elastic recoil ratio (ERR) measured at the maximum stenosed region after PTA. The ERR characterizes how much this stenosed section recoils after balloon deflation. Acting as permanent implants, stents generally reduce the diseased artery recoil by supporting its inner tissue; but the main lumen gain itself is caused by the balloon being inflated. Therefore anticipating acute clinical outcome (residual deformations, elastic recoils...) after balloon angioplasty is of pivotal importance for the evaluation of the treatment success which can influence further treatment decisions. However up to our knowledge only few Finite Element Method (FEM) studies focused on assessing outcomes at short terms after PTA alone.

Several studies have reported the development of computational models for simulating PTA in diseased arteries using FEMs. Different combinations of geometries, constitutive laws and loading conditions were implemented, affecting accordingly the resulting outcomes. In general, one key factor permitting or not FEM studies from evaluating balloon angioplasty influences on outcomes immediately after the treatment is the material model selected for modeling the diseased artery. In the lack of in vivo material characterization methods presenting a clear advantage in extracting realistic material properties of atherosclerotic arteries, current FEM studies mainly focus on in vitro based experimental data in order to develop their constitutive laws [15]. Yet, even among the limited number of available experimental studies, very few considered quantifying permanent strains in human atherosclerotic tissues and assessing their inelastic behavior at supra-physiological loadings. Accordingly, the majority of FEM studies did not include any residual deformations within their diseased arterial material models, and thus were limited in modeling PTA alone.

Most FE studies modeled the plaque and artery behavior using an elastic (linear or not) constitutive law [16-19]. This approach can be acceptable with stents being implanted within their models. If not, the simulated stenosed arteries would have theoretically fully recoiled after balloon deflation. They mainly evaluated 3D stress distributions within stenosed arteries to analyze their sensitivity to geometrical and material composition changes, or to design the deployed devices (stents). No evaluation of the balloon influence on results was proposed. Material model choice restricted these FE studies from simulating angioplasty and evaluating its outcomes directly after balloon deflation. In reality, and according to clinical findings [2023], elastic recoil immediately after PTA varies between $11 \%$ and 55\% for different stenosed regions, plaque compositions, and balloon/artery diameters ratios, the latter ratio (i.e. balloon sizing) showing highest influence on results [20].

On the other hand, some other studies did include inelasticity within their modeled systems. Some FE studies analyzed the rupture of arterial tissues by incorporating damage mechanisms without considering any permanent deformation within their models, such as: crack propagation [24], cohesive zone [25], or damage models (e.g. stress-softening: Mullins effect alone) [26]. These inelastic material models result in no residual strains at unloading, and would be limited in terms of the acute outcome evaluations after PTA as well. Other FE 
studies modeled inelasticity by including plasticity and were generally capable of assessing the immediate outcomes of an angioplasty procedure in terms of residual deformations. However, even though some presented detailed and interesting post-treatment assessments from their work [27-30], their focus was on outcomes after stent implantation and not after PTA alone.

The remaining FE studies both modeling inelasticity (including plasticity) and evaluating outcomes immediately after PTA alone (i.e. without stenting) are still limited in terms of presented results [31-35]. They assessed either the lumen gain ratio (LGR) at one location after deflation [32], the elastic recoil ratio (ERR) after deflation also at one location [33,35], or both LGR and ERR but qualitatively (no quantification presented) [31,34]. Besides LGR and/or ERR, only Holzapfel et al. presented some data on the state of deformation and/or state of stress within the artery during and after balloon angioplasty alone [31,32]. However inelasticity was modeled only within the artery by these two latter studies, while plaques were considered as rigid bodies.

Also, the few reported studies evaluating outcomes at short terms after PTA alone modeled the deployment of only one type of balloon inflated to a single sizing within a particular plaque composition. Moreover, their balloons models parameters were not estimated from specific ones which are in clinical use. Up to our knowledge, no FE study explicitly evaluated the influence of varying procedure components (balloon type, balloon sizing or plaque composition) on treatment outcomes immediately after balloon angioplasty alone. Finally, except for [33], no FE study presents acute post-angioplasty simulated outcomes in comparison with clinical findings.

The aim of the present work is to propose a generic modeling strategy of PTA, using implicit FE analysis, to simulate angioplasty treatment response at short term (i.e. right after balloon deflation), for different combinations of balloon designs, plaque material compositions, and balloon sizings in comparison with clinical results reported in the literature. The choice of implicit solver was driven by a will to control every step of the simulation without adding non-physical parameters such as artificial mass and damping. 


\section{Material and Methods}

\section{A. Balloons models}

\section{Balloons classification}

For PTA applications, a balloon must have a repeatable or controlled size (pressure vs. diameter) ensuring that it will not continue expanding and damaging the artery after opening the blockage. Knowing that there exist several high-pressure balloons in the medical industry, the aim of this section is to suggest a relation for classifying available balloons into categories based on their compliance data, to be modeled after accordingly.

Based on its material and wall thickness, each high-pressure balloon has an individual compliance chart provided by the manufacturer reflecting the pressure at which the balloon reaches its nominal diameter, in addition to its diameter change per inflation pressures [4]. Compliance is determined by either the balloon diameter change per inflation pressure step (index of stretchability (IS)), or by its diameter growth over a range of inflation pressures measured in \% (relative compliance $(R C)$ ):

Index of stretchability $=I S=\frac{D_{n+1}-D_{n}}{P_{n+1}-P_{n}}$

Relative Compliance $(\%)=R C=\frac{D_{R B}-D_{N}}{D_{N}} \times 100$

with $D_{n+1}$ and $D_{n}$ being balloon diameters at any two consecutive pressure levels $P_{n+1}$ and $P_{n}$, ranging from the unfolded inflation pressure $\left(P_{U n f}\right)$ to the rated burst pressure $\left(P_{R B}\right.$, threshold pressure a balloon is designed to accept without rupture [4]). $D_{N}$ and $D_{R B}$ are the balloon diameters at the nominal and the rated burst inflation pressures $\left(P_{N}\right.$ and $\left.P_{R B}\right)$ respectively [5,36,37]. The working pressure range provided by the manufacturer is $\left[P_{N}, P_{R B}\right]$. The unfolded diameter $\left(D_{U n f}\right)$ corresponds to the diameter reached by the balloon when pressure starts being applied prior to any inflation (i.e. at $P_{U n f}$ ). It is different to the folded diameter $\left(D_{f}\right)$ that corresponds to the inner diameter of the catheter in which the angioplasty balloon is crimped before insertion into the patient vasculature.

According to clinicians and manufacturers, there exist three general categories for characterizing available high-pressure balloons for PTA: compliant, semi-compliant, and noncompliant $[4,5]$. Conforming to areas of least resistance during inflation, high-compliance balloons are typically used in presence of soft un-calcified lesions. Differently, keeping their defined diameters at high pressures, low-compliance balloons are typically inflated within highly calcified (stiff) lesions and/or for stents full expansion post-dilations [4,5,36-38]. Lately, there has been some debate on what type of balloon material is optimal for PTA. Some retrospective observational studies suggest differences in angiographic results and overall procedural outcomes for different balloon materials [39]; others do not [5,40].

This work focuses first on modeling the main balloon families covering most balloon designs used by clinicians during PTA. In what follows, and because they would be applied the same modeling method, both compliant and semi-compliant balloons are considered belonging to 
the same category of semi-compliant balloons. Accordingly, two balloon families are studied: Semi-compliant vs. Non-compliant. In this work, all balloons data are provided by Medtronic APV, Santa Rosa CA, USA.

\section{a. Semi-compliant balloons}

Relative compliance of semi-compliant balloons made of high density polyethylene, nylon or polyolefin copolymer (POC) ranges between $10-20 \%$ [4,5] or is greater than $8 \%$ [41]. Average index of stretchability (IS) is typically around $0.095 \mathrm{~mm} / \mathrm{atm}$ for these balloons [5]. These balloons are rarely inflated within stiff stenosis to avoid the 'dog-bone effect' that would occur around the lesion at high inflation pressures [38]. Table 1 presents an example of Pressure-Diameter (P-D) compliance chart of a $2.8 \mathrm{~mm}$ semi-compliant balloon (graphed in Figure 1) in addition to its average index of stretchability.

\section{b. Non-compliant balloons}

The relative compliance of highly non-compliant balloons is generally less than 7\% [41] or $10 \%$ [5]. They are usually made of polyethylene terephthalate (PET) material. Their average index of stretchability (IS) fluctuates typically around $0.01 \mathrm{~mm} / \mathrm{atm}$ [5]. These balloons impose their own geometries to the stenosis and hence experience negligible changes in profile during high-pressure inflation [38]. For comparison with semi-compliant balloons, Table 1 also shows the P-D compliance chart for a $2.8 \mathrm{~mm}$ non-compliant balloon with its corresponding average index of stretchability. Figure 1 presents the pressure vs. diameter diagram of the non-compliant balloon as well.

Table 1: P-D compliance charts with their calculated index of stretchability for two different families of $2.8 \mathrm{~mm}$ balloon designs (non-compliant vs. semi-compliant). Green and blue cells indicate diameters at nominal and rated burst pressures $\left(P_{N}\right.$ and $\left.P_{R B}\right)$ respectively.

Figure 1: Pressure-diameter curves of the semi-compliant and non-compliant balloons.

For a more consistent categorization, balloons can be classified based on $R C$ values being related to their indices of stretchability, nominal diameters, in addition to nominal and rated burst pressures. $R C$ can hence be expressed as:

$R C=\frac{\left(P_{R B}-P_{N}\right) \times \overline{I S} \times 100}{D_{N}}$

where $\overline{I S}$ is the average value of $I S$. In this work we consider balloons with $R C$ ranging below $8 \%$ belong to the non-compliant family, whereas balloons with $R C$ above $8 \%$ belong to the semi-compliant family.

\section{Balloons material modeling}

As mentioned, an angioplasty balloon is initially crimped into the catheter body during manufacturing so to facilitate its insertion into patient vasculature. Accordingly, it unfolds first with complex kinematics (see for instance Geith et al. (2019) for details [42]) during the initial phase of the inflation process reaching eventually its unfolded diameter $\left(D_{U n f}\right)$. After 
that, any further increase in the applied inner pressure would start the balloon inflation until reaching the desired diameter with a typical behavior corresponding to its design. To limit the computational cost of our simulation, the modeling of the detailed unfolding process is neglected in this work; instead a cylindrical model is used in order to mimic the overall balloon catheter mechanical response. The appropriateness of using a cylindrical tube to simplify angioplasty simulations has been verified by Grogan et al. [43].

Our modeling strategy therefore consists of considering first a realistic folded diameter $\left(D_{f}\right)$ so that the balloon can be inserted into a highly stenosed artery, and then use the balloon compliance chart to build a constitutive law that can describe the balloon behavior mainly within its working range (between $D_{N}$ and $D_{R B}$ ). In this study, all applications are performed with $2.8 \mathrm{~mm}$ nominal diameter balloons (seen in Table 1). This rather small diameter compared to those usually used for femoral arteries PTA, was chosen to limit the computational cost of simulations at this stage of the study, that is developing the model and evaluate its validity.

For a $3 \mathrm{~mm}$ artery stenosed by a plaque of $60 \%$, any balloon catheter with a $D_{f}$ less than 1.2 $\mathrm{mm}$ can be introduced without colliding with the plaque. Regardless of balloon type, $D_{f}$ was fixed here to $1.15 \mathrm{~mm}$. It is in accordance with balloon profile values found on commerciallyavailable angioplasty balloon catheters designed by medical device manufacturers and used by clinicians.

$D_{\text {Unf }}$ was determined by plotting the P-D diagram using the manufacturer compliance charts (Figure 1). It is defined as the intercept with the diameter (ordinate) axis of the P-D graph: the diameter at approximately zero (very low) pressure. In this study, the estimated unfolded diameters of the semi-compliant and the non-compliant balloons were $D_{U n f}=1.887 \mathrm{~mm}$ and $D_{\text {Unf }}=2.740 \mathrm{~mm}$ respectively.

\section{a. Semi-compliant balloon modeling}

Having thin walls $(0.035 \mathrm{~mm})$ and a high index of stretchability, semi-compliant balloons were driven by pressure, to accurately simulate the plaque/balloon interaction, especially the dog-bone effect. Therefore, an accurate constitutive law for the balloon is required. The deployment of PTA balloons is divided into two phases: the unfolding phase (from $D_{f}$ to $D_{U n f}$ ), followed by the inflating phase (from $D_{U n f}$ to $D_{n}$ ). During the unfolding phase, the folded balloon deploys freely at very low pressures. Then, the unfolded balloon continues by an elastic deformation during the inflating phase until reaching the desired diameter $\left(D_{n}\right)$ within its working range.

In order to simplify this 2-phase deployment process, an Ogden $1^{\text {st }}$ order hyperelastic constitutive model was implemented in this work. This model is able to replace both phases by a continuous non-linear elastic expansion. From the manufacturer compliance charts (Table 1), a corresponding stress-strain $\left(\sigma_{n}, \varepsilon_{n}\right)$ curve for the semi-compliant balloon was determined using equations (4) and (5) and considering it as a thin walled pressure vessel:

$\sigma_{n}=\frac{P_{n} \times D_{n}}{2 \times t}$ 
$\varepsilon_{n}=\frac{D_{n}-D_{f}}{D_{f}}$

where $t$ is the balloon wall thickness, considered constant. Figure 2 presents the stress-strain couples computed at several pressures during the semi-compliant balloon inflation in addition to their corresponding Ogden $1^{\text {st }}$ order fit. Using these data-points and their curve fit, the Ogden law could be identified. In this work, curve-fitting computed the following parameters for the balloon Ogden law: $\mu=1.72 \mathrm{MPa}, A_{1}=4.55$ and $D_{1}=0$ and reproduces very accurately manufacturers data especially in the balloon working range.

Figure 2: Stress-strain pairs (blue crosses) in addition to their fit (red solid line) by the hyperelastic constitutive model considered for the semi-compliant balloon of this study (Table 1). $D_{f}, D_{U n f}, D_{N}$ and $D_{R B}$ are the folded, unfolded, nominal and rated-burst diameters respectively with their corresponding stress-strain values. Balloon working range is shown within the grey dashed rectangle (between $D_{N}$ and $D_{R B}$ ).

\section{b. Non-compliant balloon modeling}

During inflation of these balloons, the initial unfolding process is usually followed by a very stiff (non-compliant) response at higher pressures. Gasser and Holzapfel (2007a) reported that this irregular mechanical behavior cannot be attained using simple isotropic material models for representing the balloon [32]. Instead they suggested using the theory of fiber-reinforced materials for the design of these models.

However, having thicker walls $(0.15 \mathrm{~mm})$, negligible changes in their profiles during inflation [38] and low IS, another option for simulating non-compliant PTA balloons without losing fidelity is to drive them by radial displacement. This assumption is defendable by the fact that such balloons are stiff enough to impose their own shapes to the diseased region while inflating [31,43]. Accordingly, the inflation and deflation of non-compliant balloons were driven by radial displacement in this work.

Displacement driven simulations allow of course a perfect control of the balloon diameter evolution as well as better simulation robustness. Being fully simulated by displacement boundary conditions, there is no need for an accurate constitutive law for this balloon family.

\section{Balloons geometry, mesh and boundary conditions}

The balloon geometry is a $13 \mathrm{~mm}$ long hollow cylinder, with 2 symmetrically lofted caps at its ends for the semi-compliant family and without the caps for the non-compliant one. The balloon working length was chosen to exceed the plaque length according to manufacturers' recommendations. Balloons were meshed by 4-nodes shell elements.

For semi compliant balloons, hydrostatic fluid elements were implemented to simulate the pressure driven inflation. Hydrostatic fluid elements are usually used to model fluids that are fully enclosed by solids [44]. An incompressible fluid with a negligible thermal expansion was defined for the fluid material model used. A uniform time dependent pressure was then applied at the pressure node, located at the center of the fluid volume. This pressure varied from zero to the desired inflation pressure and then back to zero. 
Regarding the balloon design test (semi-compliant vs. non-compliant), both simulated balloon families were inflated up to their nominal diameters $\left(D_{N}=2.8 \mathrm{~mm}\right)$, and then deflated back to their initial positions. In a similar way, the semi-compliant balloon was also driven up to $D_{N}=$ $2.8 \mathrm{~mm}$ (at $P_{N}=10 \mathrm{~atm}$ ) for the different plaque material composition simulation tests. Finally, and being also interested in the assessment of balloon sizing influence on acute PTA outcomes, three different inflating pressures were then simulated using the semi-compliant balloon as well. The chosen balloon sizings with their corresponding rated diameters at each inflation pressure are depicted in Table 2. All simulated balloon diameters fall within the clinically recommended range of balloon sizings (inflated balloon diameter to healthy arterial lumen diameter ratio) of [0.9 to 1.1] for PTA treatments [5].

Table 2: Balloon sizing at each simulated inflation pressure.

Boundary conditions were defined to ensure free radial expansion of the balloons: fastened on one side (mimicking the bonding to the catheter shaft), while preventing only axial displacement at the other end (modeling the role of the tip on the balloon) for the semicompliant balloon type (Figure 3), whereas clamped on both sides for the non-compliant one.

Figure 3: Balloon catheter main components.

\section{B. Arterial and plaques models}

\section{Constitutive laws}

Even though hyperelasticity can be a good approximation for several soft tissues behaviors [15], this type of constitutive law does not allow for differentiating loading and unloading paths. Neglecting inelasticity and modeling hyperelasticity alone would prevent PTA simulations from evaluating acute treatment outcomes that usually show residual deformations [20-23]. Therefore plasticity was incorporated in the materials constitutive laws of the present work for the purpose of assessing angioplasty procedure outcomes in terms of permanent deformations.

\section{a. Plaque}

To achieve a permanent deformation in the plaque and prevent a $100 \%$ recoil, the plaque was modeled using a bilinear isotropic hardening model [45]. Material parameters for plaques were obtained from Maher et al. (2011) work [46] that experimentally reported the inelastic behavior of human atherosclerotic plaques under cyclic radial compressive loading regimes. Lipid and calcified plaques were considered in the calibration. Material coefficient for the plaques elastic behavior, i.e. dynamic stiffness and yield strength values, were attained from Maher's stress-strain experimental data corresponding to the maximal strain values after which permanent deformations start to be detected. Subsequently, the remaining inelastic 
material parameters (tangent moduli), were empirically tuned to attain the experimentally observed residual strains for given peak strains for each plaque type. The lipid and the calcified plaques material parameters are presented in Table 3; their stress-strain plots are shown in Appendix A.

Concerning plaque types simulated in this work, since a wide variability in plaque composition exists among patients, five different combinations were modeled: a homogenous lipid plaque, a homogenous calcified plaque, and three heterogeneous plaques of diverse lipid-to-calcified plaque constitution ratios (30\%, 50\% and $70 \%$ randomly distributed calcifications). All five plaque combination cases were incorporated in the parametric study of plaque material constitution influence on angioplasty outcomes. Differently, the two homogenous plaques were simulated for the balloon design and balloon sizing studies.

\section{b. Artery}

The vast majority of FE studies modeling the artery consider it as hyperelastic. Some papers modeled it as an isotropic hyperelastic material using either the Neo-Hookean [47], the Ogden [30], or the Mooney-Rivlin [18] models. Others considered incorporating anisotropy [48] to the non-linear hyperelastic material, mainly using Holzapfel-Gasser-Ogden (H-G-O) model with dispersed families of collagen fibers [49]. In this work, the artery behavior was first modeled using a Neo-Hookean model with initial shear modulus $\mu=0.227 \mathrm{MPa}$ and incompressibility parameter $D=0.177 \mathrm{MPa}^{-1}$ obtained from Paini et al. in-vivo study [50].

Then an intermediate simulation was set up to verify if these parameters provided realistic results. The intermediate simulation consisted in simulating the inflation of a semi-compliant balloon into a stenosed artery, as described in Section 3, for a lipid plaque and a calcified plaque separately. Then the simulation outcomes were compared to clinically reported data to evaluate if the constitutive law for the artery required further development. Indeed, the average loss of luminal diameter at max stenosed regions computed immediately after PTA from the intermediate simulations were $52 \%$ and $54 \%$ for the lipid and calcified plaque cases respectively. The average luminal diameter loss, also referred to as the diametric elastic recoil ratio (ERR), is computed as the luminal diameter difference between the max balloon inflation state vs. after its deflation. With the diametric ERR resulting from the intermediate simulations exceeding the clinically expected ones (30\% and 35\% respectively assumed for the lipid and calcified plaque cases as average values from clinical outcomes [20-22]), this indicates that our diseased artery in these intermediate simulations elastically recoiled more than what was observed clinically.

Two possible corrective suggestions could be considered. The first option would be to neglect the plaques material parameters calibrated according to Maher et al. experimental work as described in section 1.a, and adapt instead their constitutive laws so that ERRs of $30 \%$ and $35 \%$ would be obtained respectively in each case with residual deformations occurring only in the plaques. However, as arterial tissues has also experimentally shown an inelastic material behavior under supra-physiological loadings [51], in this work a second option was chosen: it was hypothesized that the artery itself would also experience permanent deformations; consequently it was modeled as a bilinear isotropic hardening model as well. Regarding its 
inelastic material parameters identification, the arterial wall max circumferential deformations reached in the intermediate simulations were first computed; accordingly the arterial plastic material coefficients were then tuned to satisfy the two clinically obtained ERRs of $30 \%$ and $35 \%$ after PTA for each of the lipid and the calcified plaque cases respectively. Different to the plaque that was tuned under compressive loading, the constitutive law of the artery was calibrated in tension. The obtained arterial material parameters corresponding to each of the lipid and the calcified plaque cases are shown in Table 3. Their stress-strain plots are presented in Appendix A.

Table 3: Material parameters of the lipid and calcified plaques and their corresponding arteries.

\section{Geometry, mesh and boundary conditions}

The artery was modeled as a $25 \mathrm{~mm}$ long hollow cylinder, a healthy lumen diameter of $3 \mathrm{~mm}$ and a thickness of $1 \mathrm{~mm}$. Dimensions were obtained from measurements made by Karimi et al. [52]. The modeled plaque was designed non-axisymmetric to represent irregular shapes experienced in reality. Its length was $10 \mathrm{~mm}$. Plaques are usually characterized by luminal stenosis which severity is expressed as a portion of the initial lumen [53]. Stenosis severities are commonly assessed either in terms of diameters or in terms of areas. In this work we use the diametric definition, corresponding to:

$\%$ stenosis $=\frac{D_{h l}-D_{S}}{D_{h l}} \times 100$

where $D_{h l}$ and $D_{s}$ are the healthy lumen and stenosis diameters respectively. A plaque of maximum $60 \%$ stenosis was generated.

After a mesh sensitivity analysis, both artery and plaque were meshed respectively by 178856 and 36692 4-node hybrid solid elements (of mixed u-P formulations), corresponding to typical element sizes of $0.25 \mathrm{~mm}$ vs. $0.2 \mathrm{~mm}$. The mesh-size convergence analysis is detailed in Appendix B. Meshes were connected between the two geometries at their shared circumferential region. The 3D FEM model of the complete PTA system is shown in Figure 4.

In-plane (x-y plane) arterial translations were allowed at both artery ends to allow its free adaptation to balloon inflation (preventing any unrealistic bending), the balloon being itself clamped as described in section 3. Axial translation and all rotations were prevented in the model. The non-linear contact between the balloon, the plaque, and the artery is frictionless and formulated by pure penalty. ANSYS implicit commercial finite element software (ANSYS ${ }^{\circledR}$ Academic Research Mechanical, Release 19.1.) was used to solve these largedeformation analyses, solved under the finite strain theory.

Figure 4: Finite element model of the complete percutaneous transluminal angioplasty system. The plaque represented here is heterogeneous with $50 \%$ of calcifications. 


\section{Analysis of results}

Simulation results were analyzed in terms of the elastic recoil ratio (ERR) and the lumen gain ratio (LGR) obtained after PTA for the three studies: balloon design, plaque material composition, and balloon sizing influences on acute post-procedural outcomes. The elastic recoil ratio was assessed as the difference between the max-inscribed-sphere diameter that can be generated along the centerline at max balloon inflation vs. after balloon deflation (equation (7)). In a similar way, the lumen gain ratio was calculated as the difference between maxinscribed-spheres diameter after balloon deflation vs. before balloon inflation (equation (8)). Vascular Modeling Toolkit (www.vmtk.org) was used for centerlines and max-inscribedspheres extractions. ERR is comparable to what clinicians measure after performing PTA as a control for their treatment outcomes. Figure 5 presents an illustration of the inner luminal geometries showing their centerlines and the max-inscribed-spheres appearing in two different positions along the stenosed artery: (a) before inflating the balloon, (b) at its max inflation state and (c) after its deflation.

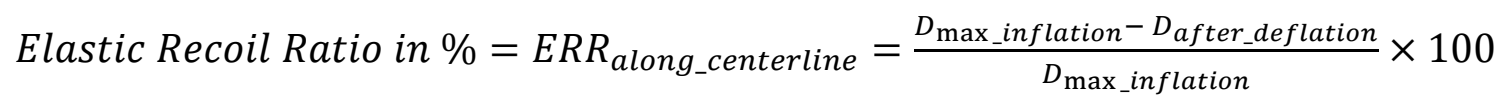

Lumen Gain Ratio in $\%=L G R_{\text {along_centerline }}=\frac{D_{\text {after_deflation }}-D_{\text {before_inflation }}}{D_{\text {before_inflation }}} \times 100$

Figure 5: Reconstructed surface geometries that correspond to the stenosed artery inner lumen showing maxinscribed-spheres (in tan) at two random sections (in red) along their centerlines for the three states of interest during balloon angioplasty simulations. 


\section{Results}

\section{A. Balloon model}

Prior to their inflation within the stenosed arteries, the two classified balloon families were independently tested.

Driven by radial displacement, the non-compliant balloon model always reaches required inflation diameters accurately. This model is robust and converges easily with a fast simulation time on a normal computer: less than one minute on an $\operatorname{Intel}(\mathrm{R}) \operatorname{Xeon}(\mathrm{R})$ workstation with 64 bit CPU dual core processors of $2.10 \mathrm{GHz}$ clock speed. Maximum circumferential deformation simulated in this balloon model was around $165 \%$.

Driven by pressure, the semi-compliant balloon model was tested for its accuracy before being inflated inside stenosed arteries. The identified set of Ogden law coefficients used in the balloon material model here reproduces very accurately manufacturers data in terms of pressure-diameter values (see working range on Figure 2) and ensures robustness, i.e. simulation convergence no matter the plaque stiffness, despite large deformations and contacts. The maximum circumferential deformation simulated with this balloon model was around $225 \%$ at its rated burst pressure.

Table 4 presents a comparison between the experimental diameter extracted from manufacturers P-D compliance charts and the FE simulated inflation diameter for the semicompliant balloon. Differences in diameters were then computed using the relative error general equation which showed negligible error values at respective inflation pressures. The simulation convergence time was around 2 minutes for this semi-compliant balloon type.

Table 4: Relative \% error between experimental and FE simulations diameters obtained at respective pressures during inflation of the semi-compliant balloon described in section a. Green and blue cells also indicate diameters at nominal and rated burst pressures $\left(P_{N}\right.$ and $\left.P_{R B}\right)$ respectively.

\section{B. Simulation of the angioplasty}

Several combinations of balloon designs, plaque material compositions, and balloon sizings were simulated. Their convergence time did not exceed 2.5 hours when simulated on the same computer, with shorter times for displacement-driven non-compliant balloons.

\section{Balloon design influence on PTA outcomes}

Each balloon design was separately simulated to its nominal diameter $\left(D_{N}=2.8 \mathrm{~mm}\right)$ within the two homogeneous plaque compositions (lipid and calcified). Figure 6(A) illustrates the plastic deformations occurring in the calcified diseased artery model after balloon deflation in an axial and a longitudinal cut view. Residual deformations were observed in both the artery and the plaque, with more significant concentration within the stenosed region.

Figures 6(B-I) and 6(B-II) respectively show the semi-compliant vs. the non-compliant balloons profiles at max inflation within the calcified plaque. It can be seen that only semi- 
compliant balloons experienced profile change during inflation, with more variations within calcified plaques (dog-bone effect). At the stenosed region, the semi-compliant balloon shifted more towards the opposite side of the plaque, causing more residual deformation in the artery opposing the stenosis. Differently, more permanent deformation was observed in the artery covering the plaque with the non-compliant balloon.

Figure 6(C) presents the balloons diameter evolution at their central planes along the inflation/deflation phases for four different cases: a non-compliant balloon, a semi-compliant balloon outside a stenosed artery, and a semi-compliant balloon inside a stenosed artery with a lipid vs. a calcified plaque. The non-compliant balloon and the semi-compliant one simulated outside the stenosed artery virtually reached their anticipated nominal diameters $\left(D_{N}\right)$ of 2.8 $\mathrm{mm}$ at max inflation. Even though the semi-compliant balloons did not reach the exact desired diameter at nominal pressure when inflated within stenosed arteries, the differences were not significant.

Figure 6(D) shows the lumen gain and the elastic recoil ratios attained along the artery axis after PTA using the two modeled balloon designs, within the lipid and calcified homogenous plaques. Figure 6(D-I) also shows the initial lumen diameter evolution along the axis. LGR directly evaluates the lumen expansion caused by the balloon inflation, thus residual strains occurrence within the tissues. ERR appraises how much the diseased artery recoils after being expanded by the balloon; if the tissues were fully elastic, they would move back to their initial positions after balloon deflation. Both LGR and ERR were larger where there was large stenosis (small initial lumen diameter), while they reached 0 or even (slight) negative values where no plaque was present. Negative values were probably caused by the surrounding elastic and plastic strains that can influence the arterial diameters locally even at healthy regions. As expected, in largely stenosed regions, ERR were higher within the calcified plaque cases (Figure 6(D-II)); consequently, lower LGR were observed within these calcified stenosis cases (Figure 6(D-I)). Finally, upon comparing the balloon-type influence on acute PTA post-procedural outcomes (LGR and ERR), no differences were observed within both simulated homogeneous plaques whether a semi-compliant or a non-compliant balloon model was used.

\footnotetext{
Figure 6: (A) Plastic deformations resulting after PTA in one simulation at $10 \mathrm{~atm}$ with red arrows representing the final lumen diameter ( $D_{\text {after_deflation }}$ ) attained after balloon deflation at 3 random positions. (B) The semi-compliant vs. the non-compliant balloons profiles at their max inflation states within a calcified stenosis. (C) Balloons diameter evolution at their central plane along the inflation and deflation process for 4 different cases: non-compliant balloon, semi-compliant balloon outside a stenosed artery, and semi-compliant balloon inside the stenosed artery by a calcified vs. a lipid plaque. (D) Lumen gain ratios (D-I) in addition to the elastic recoil ratios (D-II) obtained along the artery axis after PTA using the two different balloon designs, within the lipid and calcified homogenous plaque compositions.
}

\section{Plaque material composition influence on PTA outcomes}

To evaluate the influence of the plaque material on the PTA outcomes, the semi-compliant balloon was inflated up to its nominal diameter into a stenosed artery for different plaque compositions. Besides the two homogeneous (lipid and calcified) plaques, three heterogeneous cases of different lipid-to-calcified plaque ratios (30\%, 50\% and $70 \%$ 
randomly distributed calcifications) were also modeled for this parametric study. Upon analyzing the attained LGR and ERR values after PTA (Figure 7), it can be seen that they varied in the opposite direction with respect to the change in calcification concentration in the plaque. LGR reached its highest value ( $62 \%)$ when the plaque contained $0 \%$ of calcifications (all lipidic); it decreased progressively with the growth of calcification proportion within the plaque, reaching ( $51 \%)$ when the plaque was all calcified. Differently, the ERR had its lowest value ( $\sim 29.5 \%$ ) when the plaque had no calcifications; it gradually increased with the augmentation of calcification ratio within the plaque, reaching its highest value ( 33.5\%) when the plaque was totally calcified. Furthermore, the rate at which the LGR changed between the entirely calcified plaque and the completely lipidic one (51 to 62\%) was larger than the rate at which the ERR changed between the same plaque compositions (33.5 to 29.5\%).

Figure 7: Lumen gain ratios (A) in addition to the elastic recoil ratios (B) attained along the artery axis after PTA for different plaque material compositions (lipid-to-calcified constitution ratios), using the semi-compliant balloon inflated at $10 \mathrm{~atm}$. (A) also presents the initial lumen diameter progression along the stenosed artery prior to balloon angioplasty.

\section{Balloon sizing influence on PTA outcomes}

Balloon sizing was evaluated by simulating the semi-compliant balloon within the two homogenous plaques for different balloon diameters falling within the clinically recommended range of balloon/artery diameters ratios. Figure 8(A and B) respectively present the evolutions of LGR and ERR along the artery axis for the three driven balloon sizes. Unlike LGR that directly relates to the lumen expansion due to balloon inflation, the interpretation of ERR can be slightly more challenging when it comes to comparing balloon sizings. We can see on Figure 8(A and B) that both LGR and ERR are in positive correlation with balloon sizing, with significantly larger rates of change for the LGR in comparison to the ERR from one sizing to another. This indicates that increasing the balloon sizing to a small extent increases the ERR, and to a larger extent increases the LGR. It also means that the stenosed vessel experiences more of both plastic and elastic strains with the increase of balloon sizing, with much larger potentials of residual deformations, i.e. lumen expansion.

Figure 8(C) depicts in longitudinal cut views the max principal strain distribution in the arterial wall at max inflation during PTA for the three balloon sizings. The peak value showed significant increase at larger pressures and seems to occur at a similar location among the different sizings, within the max stenosed region.

Figure 8: Lumen gain (A) and elastic recoil (B) ratios evolution after PTA along the artery axis for diverse sizings of the semi-compliant balloon at 10,12 and $14 \mathrm{~atm}$, within the two homogenous plaque compositions (lipid and calcified).

(C) Max principal strain field in the arterial wall (in cross sectional views) during PTA at max balloon inflation for the three different balloon sizings. Max value site is also shown for each sizing. The plaque is not shown. 


\section{Discussion}

The assessment of clinical outcome after balloon angioplasty is important for the treatment success evaluation. The present paper aims at proposing a generic modeling strategy of PTA using an implicit FEM solver, to evaluate the acute post-procedural outcomes for different balloon designs, plaque material compositions, and balloon sizings. Elasto-plastic material models were implemented within both the plaque and artery to promote permanent deformations. Besides a modeling strategy for both semi- and non-compliant balloons, results were mainly assessed in terms of LGR and ERR. Maximal principal strains within the arterial walls were also evaluated for the different balloon sizings simulated.

Based on their compliance characteristics, balloons were categorized into non-compliant and semi-compliant families. Classification was performed using a relation we developed linking known relative compliance ranges $[4,41]$ to indices of stretchability, nominal diameters, in addition to nominal and rated burst pressures, which were extracted from the balloons compliance charts. This relation is essential for overcoming naming incongruities that might occur among different users.

Each balloon type was first modeled independently. Based on the hypothesis that they are stiff enough to inflate within the stenosed region with minor changes in their profiles [31,38,43], non-compliant balloons inflation was driven by displacement; thus no accuracy verification was required. Differently, driven by pressure, semi-compliant balloons were tested first outside the stenosed artery for accuracy. Existing FE studies used isotropic elastic materials as well to model their cylindrical geometries in order to represent PTA semi-compliant balloon inflation. Decorato et al. (2014) for example used a linear elastic model [35], while Schiavone and Zhao (2015) used a hyper-elastic Mooney-Rivlin one [54], both inflating their balloons by internal pressure. However, material parameters were not estimated from specific balloons in clinical use and/or did not present any comparison with real balloon inflation (P-D) data to verify the accuracy of their balloon models alone. In this work, the implemented Ogden law did provide high accuracy in mimicking the semi-compliant balloon deployment process when compared to P-D manufacturer data, specifically within its working range (very low relative \% errors as seen in table 4). Moreover, convergence times are satisfyingly small, with inflations starting from realistic folded diameters (as manufactured), capable to be introduced and simulated inside large-enough stenosis for typical PTA treatments.

The methodology was also applied on different balloons and resulted in similar negligible errors which validate our balloon model. However for very unfavorable cases such as large arteries and thick plaques leading to the use of long balloons with small diameter $\left(D_{f}\right)$, the identified value of $\mu$ can be too small to ensure robustness due to insufficiently large tangent stiffness at the beginning of the inflation, between $D_{f}$ and $D_{U n f}$. In that case, we suggest identifying Ogden law after removing the first two points of the manufacturers data (below the nominal pressure), to obtain a larger value of $\mu$. The cost would be a slightly poorer accuracy in the working range but it improves robustness.

In order to analyze their influence on outcomes at short term after balloon angioplasty, the two modeled balloon designs were simulated within stenosed arteries. This required a 
preliminary material calibration for both the plaque and artery. The obtained parameters allow the computation of realistic ERRs. Even though the calcified plaque type simulated in our work is relatively soft compared to the stiff micro calcifications reported by the experimental work of Ebenstein et al. [55], it still falls within the stiffness ranges reported by other experimental studies for calcified plaques as well, such as [46,56,57] for example. It should be noted that a large variability of data was shown among different studies reporting plaque stiffness in the literature. Besides the natural variability of the plaque behavior, this might be caused by the different techniques used to measure the mechanical properties of plaques including unconfined compression, micro-indentation, and nano-indentation. However, in this work we focused on studies that applied dynamic loading conditions to present the inelastic and recoverability behavior of plaques. With only few experimental studies in the literature reporting the inelastic behavior of atherosclerotic plaques, the work of Maher et al. [46] (which assessed the global mechanical properties of plaques under supra-physiological unconfined cyclic compressive loading) was favored in our study.

When simulating PTA, as expected, only the semi-compliant balloon showed a change in its profile during inflation, with a larger alteration inside the calcified stenosis (Figure 6(B)). This phenomenon is known as the 'dog-bone effect' (small waist appearance in middle of balloon), which usually occurs around stiff plaques when inflated with softer balloons [37,38]. This uncontrolled over-expansion of the balloon outside the plaque may damage the artery or cause balloon rupture; therefore, clinicians usually suggests using the non-compliant balloons in the presence of calcified lesions for more effective dilations $[4,37,38]$. Because of the plaque resistance, semi-compliant balloons do not exactly reach their expected diameter when inflated into a stenosed artery, but this is not significant (Figure 6(C)). For both soft and stiff plaques, negligible differences were also observed in terms of the LGR and ERR obtained after PTA upon changing balloon design (Figure 6(D)). Therefore, in this work, balloon type showed no influence on acute post-procedural outcomes after PTA. This finding is in accordance with the retrospective nonrandomized studies that showed no difference between semi-compliant and non-compliant balloons with respect to the final procedural outcomes (e.g. final residual stenosis) [5,40].

Independent from the balloon design, plaque composition or balloon inflation size used, LGR and ERR always showed a negative correlation to the initial lumen diametric evolution along the artery axis. This indicates that as expected, the most stenosed regions (smallest initial lumen diameters) are the most susceptible to balloon angioplasty treatments. It explains why clinicians usually assess PTA outcomes at such regions as well [4,20,21].

When interpreting the ERRs attained from our simulations in comparison to clinically reported values [20-22], it confirms that the assumption for the material models of the artery and plaque considered in this work is reasonable. Hanet et al. found mean clinical recoil ratios of $32 \% \pm 12 \%$ of luminal diameter immediately after PTA in coronary arteries [21]. Similarly Isner et al. and Gardiner et al. reported that elastic recoils in iliac peripheral arteries respectively averaged $28.6 \% \pm 7.2 \%$ vs. $36 \% \pm 11 \%$ [20,22]. Clinicians generally compute their ERR at most stenosed regions. With our measured ERR values at similar locations ranging between $28 \%$ and $36 \%$ for the different balloon designs, plaque material 
compositions, and balloon sizings simulated, our results showed consistency with clinical findings. This 28-36\% range of ERR can appear rather narrow, this is due to the fact that it is measured at locations with large stenosis (60\%); a wider range would be obtained when considering different locations with different degrees of severity.

Unlike balloon types, plaque material composition showed an impact on simulated results obtained immediately after PTA (LGR and ERR). As expected, following the calibration we applied to the artery and the plaque (section B), the calcified plaque case experienced a higher ERR compared to the lipid one. This assumption was based on clinical findings that reported rather higher ERR values in the presence of calcified plaques with the hypothesis that these stiff plaques would experience less compression themselves while expanding more the elastic arterial wall during reasonably sized inflations, leading to higher recoils after balloon deflation, even if the differences were not major [20,21]. On the other hand, LGR decreases while calcification proportion within the plaque increases. This indicates that plastic deformations would be higher when the plaque is less calcified. Both ERR and LGR were affected by the calcification ratio in the plaque but without a significant effect of the randomized distributions of components themselves.

Balloon sizing has also a significant influence on results attained shortly after balloon angioplasty. Both ERR and LGR showed a positive correlation to the augmentation in inflation pressure, independently from the plaque composition. This finding is in agreement with clinical observations stating that the elastic recoil is generally in direct association with the balloon/artery ratio [20,21,23]. However ERR and LGR do not evolve at the same rate with respect to sizing. Indeed, at the max stenosed region, for pressure growing from 10 to 14 atm, the ERR rose from $29.5 \%$ to $30.5 \%$ with the lipid plaque and from $33.5 \%$ to $35.5 \%$ with the calcified one, while the LGR rose from $62 \%$ to $83 \%$ with the lipid plaque and from $51 \%$ to $68 \%$ with the calcified one. This indicates that at higher sizings, the treated stenosed artery experiences significant plastic strain. In this work, we did not model the complex damage and flow mechanisms occurring at small scales in diseased arteries during PTA; plasticity was used instead to represent these phenomena. It is important to note that this damage can be always considered as a positive phenomenon allowing a larger lumen after balloon angioplasty (as 'controlled vessel injuries' [14]), until the artery itself become vulnerable to dissections.

Max principal strains occurring in the arterial walls at max inflations for the three simulated sizings were interpreted as an indication for their risk of rupture. With strain peak values showing sharp increases from 0.19 to 0.25 for a sizing change from 10 to 14 atm, the artery vulnerability to rupture is influenced by the balloon sizing choice as well. Therefore a compromise should be settled achieving the desired lumen gain after balloon angioplasty on one hand, but without dissecting the healthy arterial tissues from another.

One should keep in mind that despite continuing to be the method that all other available endovascular treatment modalities are still compared against to date [13], the standard plain balloon angioplasty technique is clinically associated to some limitations as well. Treatment of complex lesions including heavily calcified long lesions and chronic total occlusions 
remains challenging for angioplasty balloons, even for drug-eluting ones [58]. Accordingly, several interventional approaches are being lately further investigated and applied either as stand-alone treatments or as lesions preparations prior to PTA to overcome its limitations. Numerous balloons of different mechanisms of action available nowadays on the market are devoted to preparing such complex lesions [59]. Focal force balloons and endovascular atherectomy devices are examples of treatment alternatives designed to prepare complicated lesions to enhance acute post-procedural outcomes. In this context, simulating angioplasty remains an issue of interest, either because PTA is part of the treatment process, or in order to evaluate and define the interventional strategy.

Finally, limitations of this study mainly concern the artery and plaque models. In this work we do not claim to model the detailed phenomena of damage that might occur during PTA. Instead, plasticity is meant to model any complex damage and flow phenomena occurring at small scales within the diseased artery. The main reason for our choice is that we aim to present a tool that can then be easily applied on patient-specific geometries and be incorporated into the clinical routine, without the need of additional imaging. With computed tomography angiography (CTA) being a principal part of the clinical routine, it is generally known that these images resolution would not allow for a detailed differentiation of the plaque components within the diseased arteries, especially for small diameter arteries. Besides, the calibration of the proposed constitutive laws is mostly based on kinematic quantities, such as residual strains extracted from Maher's work and the clinical ERR provided by different papers; therefore, the proposed model might require further validation to assess a risk of rupture based on maximum stress.

We are also aware that modeling the artery as an isotropic single-layer tissue is a limitation, as it is known to exhibit an anisotropic behavior. However, even though in-vitro experimental studies evaluating atherosclerotic tissues behaviors are generally available in the literature, only a few among them considered studying the inelastic behavior of such tissues. Up to our knowledge, no study presented the inelastic behavior of each arterial layer separately under supra-physiological loadings. Similarly, very limited data is available regarding the inelastic behavior (in terms of residual deformation) of human arterial tissues supra-physiologically loaded in different directions as well. Accordingly, with the lack of the necessary experimental data that represent the anisotropic and/or layer specific inelastic behaviors of arteries, in this work and as a first contribution, an inelastic single-layered isotropic artery was considered and was tuned correspondingly to model residual deformations obtained directly after PTA.

Yet our proposed model showed consistent results in comparison with clinical findings; it seems to be a promising tool towards outcomes evaluation at short terms after PTA. Furthermore, the material parameters of the modeled arteries were tuned based on average ERR values of $30 \%$ and $35 \%$ for the lipid vs. calcified plaque cases respectively obtained from clinical studies. Nevertheless, a more precise assumption could be integrated later after applying our calibration procedure on various patient-specific geometries of different plaque compositions and severities. This can be performed in the presence of detailed pre- and post- 
operative clinical routine images for the simulated diseased region before vs. after balloon angioplasty.

\section{Conclusion}

This work proposes a generic modeling strategy of PTA using an implicit FEM solver capable of assessing post-procedural outcomes at short terms after balloon deflation. After classifying and modeling balloons, and developing and identifying consistent constitutive laws for both the plaque and artery, we present a parametric study based on balloon type, plaque composition and balloon sizing, which quantifies their influence on the ERR and the LGR. It shows that for a given plaque, balloon sizing is the key factor to obtain a satisfactory postprocedural lumen diameter, as it allows to control the amount of plastic strain stored in both the artery and the plaque.

Now that the framework of a relatively fast and accurate PTA modeling within a generic stenosed artery is set, the future aim of the present work is first its application to patientspecific situations, of different arterial diameters, plaque material compositions and severities. Using clinical per- and post-operative CTA data, a further validation of our model material parameters will be appraised, for future clinical use. Additionally, another future aim of the work is to generate a reduced order model from our presented generic model capable of performing similar parametric studies in even smaller times (in terms of minutes or seconds), to be integrated in clinical decision support systems.

\section{Acknowledgement}

This work was partially supported by the Bretagne region and by the National Research Agency (ANR) in the framework of 'Investissement d'Avenir Program' through Labex (ANR-11-LABX-0004).

We thank Nicolas Courtial from LTSI lab for his support on VTK files format.

\section{Appendix}

\section{A. Artery and plaque stress-strain plots}

Figure A-1 and Figure A-2 respectively present the material behaviors of the lipid and calcified plaques in addition to their corresponding arteries.

Figure A-1: Lipid and calcified plaque materials stress-strain plots.

Figure A-2: Arterial material stress-strain plot corresponding to each of the lipid and calcified plaques. 


\section{B. Mesh-size sensitivity analysis}

The artery and the plaque were meshed by 4-node hybrid solid elements in Ansys workbench. These elements have a linear displacement and a hydrostatic pressure behavior. To avoid volumetric locking, elements with mixed u-P formulations were implemented to enhance accuracy within our simulations.

Figure B-1 presents the variation of the total strain energy at max balloon inflation and the result file size with respect to different element sizes for the artery and the plaque separately. Towards limiting the simulation computational costs while keeping a satisfying accuracy, element sizes of $0.25 \mathrm{~mm}$ and $0.2 \mathrm{~mm}$ were chosen for meshing the artery and the plaque respectively. Figure B-2 shows the plastic strain distribution along stenosed arteries with different mesh size combinations; their color-maps emphasize the minor impact of mesh density on the results and justify our elements sizes choice in this work.

Figure B-1: Artery (A) and plaque (B) mesh-sizes influences on the total strain energy stored in the diseased arteries at max inflation (diameter of $3 \mathrm{~mm}$ ), and on their resulting files sizes.

Figure B-2: Plastic strain distribution along a longitudinal cross-section of the stenosed artery corresponding to different combinations of element sizes used for respectively meshing the artery and the plaque in each simulated case. 


\section{References:}

1. George SJ, Johnson J. Atherosclerosis: Molecular and Cellular Mechanisms. In: WileyVCH Verlag GmbH \& Co. KGaA, Weinheim; 2010.

2. Brown MM, Rogers J, Bland JM. Endovascular versus surgical treatment in patients with carotid stenosis in the Carotid and Vertebral Artery Transluminal Angioplasty Study (CAVATAS): A randomised trial. Lancet 2001; 357(9270):1729-1737. doi:10.1016/S0140-6736(00)04893-5.

3. Cunnane EM, Barrett HE, Kavanagh EG, Mongrain R, Walsh MT. The influence of composition and location on the toughness of human atherosclerotic femoral plaque tissue. Acta Biomaterialia 2016; 31:264-275. doi:10.1016/j.actbio.2015.11.056.

4. Baim DS. Percutaneous Balloon Angioplasty and General Coronary Intervention. In: D. Baim, ed. Grossman's Cardiac Catheterization, Angiography, and Intervention, 7th ed.; 2005:433-466.

5. Safian RD, Hoffmann MA, Almany S, et al. Comparison of coronary angioplasty with compliant and noncompliant balloons (the angioplasty compliance trial). The American Journal of Cardiology 1995; 76(7):518-520.

6. Salem HK, Gussenhoven EJ, Zhong Y, et al. Effect of Balloon Angioplasty on Femoral Artery Evaluated With Intravascular Ultrasound Imaging. Circulation 1992; 86(2):483-493.

7. Becquemin JP, Favre JP, Marzelle J, et al. Systematic versus selective stent placement after superficial femoral artery balloon angioplasty: A multicenter prospective randomized study. Journal of Vascular Surgery 2003; 37:487-94.

8. Chen SL, Guo NS, Chen GD, et al. The Immediate Elastic Recoil of Percutaneous Coronary Angioplasty and Intracoronary Stent Implantation. Journal of Practical Radiology 2001; 10:726-8.

9. Conti M, Marconi M, Campanile G, et al. Patient-specific finite element analysis of popliteal stenting. Meccanica 2017; 52(3):633-644. doi:10.1007/s11012-016-0452-9.

10. Wong G, Lahsaei S, Aoun J, Garcia LA. Management of common femoral artery occlusive disease: A review of endovascular treatment strategies and outcomes. Catheterization and Cardiovascular Interventions 2019; 93(3):514-521.

11. Diamantopoulos, A. Katsanos K. Treating Femoropopliteal Disease: Established and Emerging Technologies. Seminars in Interventional Radiology 2014; 31(4):345-352.

12. Jaff MR, White CJ, Hiatt WR, et al. An Update on Methods for Revascularization and Expansion of the TASC Lesion Classification to Include Below-the-Knee Arteries: A Supplement to the Inter-Society Consensus for the Management of Peripheral Arterial Disease (TASC II). Vascular Medicine 2015; 20(5):465-478.

13. Kansal A, Long CA, Patel MR, Jones WS. Endovascular treatment of femoro-popliteal lesions. Clinical Cardiology 2019; 42(1):175-183.

14. Castaneda-zuniga WR. Pathophysiology of transluminal angioplasty. In: Improvement 
of Myocardial Perfusion, edited by J. Meyer, R. Erberl, and H. J. Rupprecht. Boston, MA: Martinus Nijhoff. 1985:138-141.

15. Holzapfel GA, Mulvihill JJ, Cunnane EM, Walsh MT. Computational approaches for analyzing the mechanics of atherosclerotic plaques: A review. Journal of Biomechanics 2014; 47(4):859-869. doi:10.1007/s10865-016-9757-3.

16. Pericevic I, Lally C, Toner D, Kelly DJ. The influence of plaque composition on underlying arterial wall stress during stent expansion: The case for lesion-specific stents. Medical Engineering and Physics 2009; 31(4):428-433. doi:10.1016/j.medengphy.2008.11.005.

17. Kiousis DE, Gasser TC, Holzapfel GA. A numerical model to study the interaction of vascular stents with human atherosclerotic lesions. Annals of Biomedical Engineering 2007; 35(11):1857-1869. doi:10.1007/s10439-007-9357-z.

18. Auricchio F, Conti M, Beule M De, Santis G De, Verhegghe B. Carotid artery stenting simulation: From patient-specific images to finite element analysis. Medical Engineering and Physics 2011; 33(3):281-289. doi:10.1016/j.medengphy.2010.10.011.

19. Auricchio F, Conti M, Ferrara A, et al. Patient-specific finite element analysis of carotid artery stenting: a focus on vessel modeling. International Journal for Numerical Methods in Biomedical Engineering 2013; 29:645-664.

20. Gardiner J, Bonn J, Sullivan KL. Quantification of elastic recoil after balloon angioplasty in the iliac arteries. Journal of Vascular and Interventional Radiology 2001; 12(12):1389-1393. doi:10.1016/s1051-0443(07)61694-7.

21. Hanet C, Wijns W, Michel X, Schroeder E. Influence of balloon size and stenosis morphology on immediate and delayed elastic recoil after percutaneous transluminal coronary angioplasty. Journal of the American College of Cardiology 1991; 18(2):506511. doi:10.1016/0735-1097(91)90607-B.

22. J. Isner, K. Rosenfield, D. Losordo, L. Rose, R.E. Langevin, S. Razvi, and B. Kosowsky. Combination Balloon-Ultrasound Imaging Catheter for Percutaneous Transluminal Angioplasty Validation of Imaging, Analysis of Recoil, and Identification of Plaque Fracture. 1991:739-754.

23. Kimball BP, Bui S, Cohen EA, Carere RG, Adelman AG. Comparison of acute elastic recoil after directional coronary atherectomy versus standard balloon angioplasty. American Heart Journal 1992; 124(6):1459-1466. doi:10.1016/0002-8703(92)900573 .

24. Versluis A, Bank AJ, Douglas WH. Fatigue and plaque rupture in myocardial infarction. Journal of Biomechanics 2006; 39:339-347.

25. Gasser TC, Holzapfel GA. Modeling plaque fissuring and dissection during balloon angioplasty intervention. Annals of Biomedical Engineering 2007; 35:711-723.

26. Balzani D, Brinkhues S, Holzapfel GA. Constitutive framework for the modeling of damage in collagenous soft tissues with application to arterial walls. Computer Methods in Applied Mechanics and Engineering 2012; 213-216:139-151.

27. Gastaldi D, Morlacchi S, Nichetti R, et al. Modelling of the provisional side-branch 
stenting approach for the treatment of atherosclerotic coronary bifurcations: effects of stent positioning. Biomechanics and Modeling in Mechanobiology 2010; 9:551-561.

28. Conway C, Mcgarry JP, MchugCHUGH PE. Modelling of Atherosclerotic Plaque for Use in a Computational Test-Bed for Stent Angioplasty. Annals of Biomedical Engineering 2014; 42(12):2425-2439.

29. Conway C, McGarry JP, Edelman ER, Al. E. Numerical Simulation of Stent Angioplasty with Predilation: An Investigation into Lesion Constitutive Representation and Calcification Influence. Annals of Biomedical Engineering 2017; 45:2244-2252. doi:10.1007/s10741-014-9462-7.Natural.

30. Iannaccone F, Chiastra C, Karanasos A, et al. Impact of plaque type and side branch geometry on side branch compromise after provisional stent implantation: a simulation study. EuroIntervention 2017; 13:e236-e245.

31. Holzapfel GA, Stadler M, Schulze-Bauer CA. A Layer-Specific Three-Dimensional Model for the Simulation of Balloon Angioplasty using Magnetic Resonance Imaging and Mechanical Testing. Annals of Biomedical Engineering 2002; 30(6):753-767. doi:10.1114/1.1492812.

32. Gasser TC, Holzapfel GA. Finite element modeling of balloon angioplasty by considering overstretch of remnant non-diseased tissues in lesions. Computational Mechanics 2007; 40:47-60.

33. Liang DK, Yang DZ, Qi M, Wang WQ. Finite element analysis of the implantation of a balloon-expandable stent in a stenosed artery. International Journal of Cardiology 2005; 104(3):314-318. doi:10.1016/j.ijcard.2004.12.033.

34. Dalong Li; Anne M. Robertson; Guoyu Lin; and Michael Lovell; Finite element modeling of cerebral angioplasty using a structural multi-mechanism anisotropic damage model. INTERNATIONAL JOURNAL FOR NUMERICAL METHODS IN ENGINEERING, 92 2012; (June):457-474. doi:10.1002/nme.

35. Decorato I, Kharboutly Z, Legallais C, Salsac AV. Comparison of two endovascular treatments of a stenosed arteriovenous fistula: Balloon-angioplasty with and without stenting. International Journal of Artificial Organs 2014; 37(10):763-772. doi:10.5301/ijao.5000324.

36. Saab MA. Applications of High-Pressure Balloons in the Medical Device Industry; 2000. Accessed September 20, 2020, <https://www.mddionline.com/applications-highpressure-balloons-medical-device-industry>.

37. Gupta S. Overview of Balloon Catheter, Accessed November 2019, <http://www.scai.org/Assets/31e9f931-160b-459c-9d7b-

b6771621b642/635742456224670000/gupta-guidecatheters-and-guidewire-pdf> .

38. Romagnoli E, Sangiorgi GM, Cosgrave J, Guillet E, Colombo A. Drug-Eluting Stenting. The Case for Post-Dilation. JACC: Cardiovascular Interventions 2008; 1(1):22-31. doi:10.1016/j.jcin.2007.10.005.

39. Ellis SG, Topol EJ. Results of percutaneous transluminal coronary angioplasty of highrisk angulated stenoses. The American Journal of Cardiology 1990; 66(12):932-937. doi:10.1016/0002-9149(90)90928-T. 
40. Bach RG, Kern MJ, Aguirre F V., Donohue TJ, Bell C, Penick D. Effects of percutaneous transluminal coronary angioplasty balloon compliance on angiographic and clinical outcomes. The American Journal of Cardiology 1993; 72(12):904-907. doi:https://doi.org/10.1016/0002-9149(93)91104-P.

41. MEDICAL N. Medical Balloons, accessed 11 July 2020, $<$ https://www.nordsonmedical.com/Resources/Download?title=Medical\%20Balloons\& category=Sell\%20Sheets $>$.

42. Geith MA, Swidergal K, Hochholdinger B. On the importance of modeling balloon folding, pleating, and stent crimping: An FE study comparing experimental inflation tests. International Journal for Numerical Methods in Biomedical Engineering 2019; 35:e3249.

43. Grogan JA, Leen SB, McHugh PE. Comparing coronary stent material performance on a common geometric platform through simulated bench testing. Journal of the mechanical behavior of biomedical materials 2012; 12:129-38.

44. Roche D. ANSYS: ACT Hydrostatic Fluid. 2017:1-31.

45. Gaertner EL, Bortoli MGD de. Some Aspects for the Simulation of a Non-Linear Problem with Plasticity and Contact. 2006 International ANSYS Conference 2006:115.

46. Maher E, Creane A, Sultan S, Hynes N, Lally C, Kelly DJ. Inelasticity of Human Carotid Atherosclerotic Plaque. Annals of Biomedical Engineering 2011; 39(9):24452455. doi:10.1007/s10439-011-0331-4.

47. Speelman L, Akyildiz AC, DenAdel B, Wentzel JJ, et al. Initial stress in biomechanical models of atherosclerotic plaques. Journal of Biomechanics 2011; 44:2376-2382.

48. Kiousis DE, Rubinigg SF, Auer M, Holzapfel GA. A Methodology to Analyze Changes in Lipid Core and Calcification Onto Fibrous Cap Vulnerability: The Human Atherosclerotic Carotid Bifurcation as an Illustratory Example. Journal of Biomechanical Engineering 2009; 131(12):121002. doi:10.1115/1.4000078.

49. Gasser TC, Ogden RW, Holzapfel GA. Hyperelastic modelling of arterial layers with distributed collagen fibre orientations. Journal of the royal society interface 2006; 3(6):15-35. doi:10.1098/rsif.2005.0073.

50. Paini A, Boutouyrie P, Calvet D, Zidi M, Agabiti-Rosei E, Laurent S. Multiaxial mechanical characteristics of carotid plaque: Analysis by multiarray echotracking system. Stroke 2007; 38(1):117-123. doi:10.1161/01.STR.0000251796.38954.b2.

51. Maher E, Early M, Creane A, Lally DJ, Kelly C. Site specific inelasticity of arterial tissue. Journal of Biomechanics 2012; 45(8):1393-1399.

52. Karimi A, Navidbakhsh M, Faghihi S, Shojaei A, Hassani K. A finite element investigation on plaque vulnerability in realistic healthy and atherosclerotic human coronary arteries. Proceedings of the Institution of Mechanical Engineers, Part H: Journal of Engineering in Medicine 2013; 227(2):148-161. doi:10.1177/0954411912461239.

53. Sadat U, Teng Z, Young VE, et al. Association between biomechanical structural 
stresses of atherosclerotic carotid plaques and subsequent ischaemic cerebrovascular events - A longitudinal in vivo magnetic resonance imaging-based finite element study. European Journal of Vascular and Endovascular Surgery 2010; 40(4):485-491. doi:10.1016/j.ejvs.2010.07.015.

54. Schiavone A, Zhao LG. A study of balloon type, system constraint and artery constitutive model used in finite element simulation of stent deployment. Mechanics of Advanced Materials and Modern Processes 2015; 1:1-15.

55. Ebenstein DM, Coughlin D, Chapman J, et al. Nanomechanical properties of calcification, fibrous tissue, and hematoma from atherosclerotic plaques. Journal of biomedical materials research 2009; 91(4):1028-1037.

56. Lee RT, Richardson SG, Loree HM, et al. Prediction of mechanical properties of human atherosclerotic tissue by high-frequency intravascular ultrasound imaging an in vitro study. Arteriosclerosis,Thrombosis, and Vascular Biology 1992; 12:1-5.

57. Walraevens J, Willaert B, De Win G, et al. Correlation between compression, tensile and tearing tests on healthy and calcified aortic tissues. Medical Engineering and Physics Physics 2008; 30(9):1098-1104.

58. Blessing E, Lugenbiel I, Holden A. The evidence to support the use of focal force balloon technology to improve outcomes in the treatment of lower extremity arterial occlusive disease. The Journal of Cardiovascular Surgery 2019; 60(1):14-20.

59. Ettorre L, Prouse G, Giovannacci L, et al. Vessel Preparation: What is the Evidence in the BTK Segment? VASCULAR DISEASE MANAGEMENT 2020; 17(7):E142-E149. 


\section{Figure legends}

Figure 1: Pressure-diameter curves of the semi-compliant and non-compliant balloons.

Figure 2: Stress-strain pairs (blue crosses) in addition to their fit (red solid line) by the hyperelastic constitutive model considered for the semi-compliant balloon of this study (Table 1). $D_{f}, D_{U n f}, D_{N}$ and $D_{R B}$ are the folded, unfolded, nominal and rated-burst diameters respectively with their corresponding stress-strain values. Balloon working range is shown within the grey dashed rectangle (between $D_{N}$ and $D_{R B}$ ).

Figure 3: Balloon catheter main components.

Figure 4: Finite element model of the complete percutaneous transluminal angioplasty system. The plaque represented here is heterogeneous with $50 \%$ of calcifications.

Figure 5: Reconstructed surface geometries that correspond to the stenosed artery inner lumen showing maxinscribed-spheres (in tan) at two random sections (in red) along their centerlines for the three states of interest during balloon angioplasty simulations.

Figure 6: (A) Plastic deformations resulting after PTA in one simulation at 10 atm with red arrows representing the final lumen diameter $\left(D_{\text {after_deflation }}\right)$ attained after balloon deflation at 3 random positions. (B) The semi-compliant vs. the non-compliant balloons profiles at their max inflation states within a calcified stenosis. (C) Balloons diameter evolution at their central plane along the inflation and deflation process for $\mathbf{4}$ different cases: non-compliant balloon, semi-compliant balloon outside a stenosed artery, and semi-compliant balloon inside the stenosed artery by a calcified vs. a lipid plaque. (D) Lumen gain ratios (D-I) in addition to the elastic recoil ratios (D-II) obtained along the artery axis after PTA using the two different balloon designs, within the lipid and calcified homogenous plaque compositions.

Figure 7: Lumen gain ratios (A) in addition to the elastic recoil ratios (B) attained along the artery axis after PTA for different plaque material compositions (lipid-to-calcified constitution ratios), using the semi-compliant balloon inflated at $10 \mathrm{~atm}$. (A) also presents the initial lumen diameter progression along the stenosed artery prior to balloon angioplasty.

Figure 8: Lumen gain (A) and elastic recoil (B) ratios evolution after PTA along the artery axis for diverse sizings of the semi-compliant balloon at 10, 12 and 14 atm, within the two homogenous plaque compositions (lipid and calcified). (C) Max principal strain field in the arterial wall (in cross sectional views) during PTA at max balloon inflation for the three different balloon sizings. Max value site is also shown for each sizing. The plaque is not shown.

Figure A-1: Lipid and calcified plaque materials stress-strain plots.

Figure A-2: Arterial material stress-strain plot corresponding to each of the lipid and calcified plaques.

Figure B-1: Artery (A) and plaque (B) mesh element sizes influences on the total strain energy stored in the diseased arteries at max inflation (diameter of $3 \mathrm{~mm}$ ), and on their resulting files sizes.

Figure B-2: Plastic strain distribution along a longitudinal cross-section of the stenosed artery corresponding to different combinations of element sizes used for respectively meshing the artery and the plaque in each simulated case. 


\section{Tables}

Table 1: P-D compliance charts with their calculated index of stretchability for two different families of $2.8 \mathrm{~mm}$ balloon designs (non-compliant vs. semi-compliant). Green and blue cells indicate diameters at nominal and rated burst pressures $\left(P_{N}\right.$ and $\left.P_{R B}\right)$ respectively.

\begin{tabular}{|c|c|c|c|c|}
\hline \multirow{2}{*}{$\begin{array}{c}\text { Pressure } \\
{\left[\boldsymbol{P}_{n}\right]} \\
(\mathbf{a t m})\end{array}$} & \multicolumn{2}{|c|}{ Non-Compliant $2.8 \mathrm{~mm}$ balloon } & \multicolumn{2}{|c|}{ Semi-Compliant $2.8 \mathrm{~mm}$ balloon } \\
\hline & $\begin{array}{c}\text { Diameter }\left[D_{n}\right] \\
(\mathbf{m m})\end{array}$ & $\begin{array}{c}\text { Index of } \\
\text { stretchability }[I S] \\
(\mathbf{m m} / \mathbf{a t m})\end{array}$ & $\begin{array}{l}\text { Diameter }\left[D_{n}\right] \\
(\mathbf{m m})\end{array}$ & $\begin{array}{c}\text { Index of } \\
\text { stretchability }[I S] \\
(\mathrm{mm} / \mathrm{atm})\end{array}$ \\
\hline 8 & 2.80 & - & 2.60 & - \\
\hline 9 & 2.82 & $2.00 \mathrm{E}-02$ & 2.65 & $5.00 \mathrm{E}-02$ \\
\hline 10 & 2.84 & $2.00 \mathrm{E}-02$ & 2.80 & $1.50 \mathrm{E}-01$ \\
\hline 11 & 2.85 & $1.00 \mathrm{E}-02$ & 2.91 & 1.10E-01 \\
\hline 12 & 2.87 & $2.00 \mathrm{E}-02$ & 3.02 & 1.10E-01 \\
\hline 13 & 2.88 & 1.00E-02 & 3.10 & 8.00E-02 \\
\hline 14 & 2.90 & $2.00 \mathrm{E}-02$ & 3.20 & $1.00 \mathrm{E}-01$ \\
\hline 15 & 2.90 & $0.00 \mathrm{E}+00$ & 3.27 & 7.00E-02 \\
\hline 16 & 2.91 & 1.00E-02 & 3.32 & 5.00E-02 \\
\hline 17 & 2.92 & $1.00 \mathrm{E}-02$ & 3.43 & 1.10E-01 \\
\hline 18 & 2.93 & $1.00 \mathrm{E}-02$ & 3.53 & $1.00 \mathrm{E}-01$ \\
\hline 19 & 2.95 & $2.00 \mathrm{E}-02$ & 3.60 & $7.00 \mathrm{E}-02$ \\
\hline 20 & 2.95 & $0.00 \mathrm{E}+00$ & 3.70 & $1.00 \mathrm{E}-01$ \\
\hline 21 & 2.96 & $1.00 \mathrm{E}-02$ & & \\
\hline 22 & 2.97 & 1.00E-02 & & \\
\hline 23 & 2.98 & 1.00E-02 & & \\
\hline 24 & 2.99 & 1.00E-02 & & \\
\hline 25 & 2.99 & $0.00 \mathrm{E}+00$ & & \\
\hline 26 & 3.00 & $1.00 \mathrm{E}-02$ & & \\
\hline 27 & 3.01 & $1.00 \mathrm{E}-02$ & & \\
\hline 28 & 3.02 & $1.00 \mathrm{E}-02$ & & \\
\hline \multicolumn{2}{|c|}{$\begin{array}{c}\text { Averaged index of } \\
\text { stretchability }[\overline{\boldsymbol{I} S}](\mathrm{mm} / \mathrm{atm}) \pm \\
\text { Standard Deviation }\end{array}$} & $\begin{array}{c}\text { 1.10E-02 } \\
\pm 6.24 \mathrm{E}-03\end{array}$ & & $\begin{array}{c}\text { 9.17E-02 } \\
\pm 2.76 \mathrm{E}-02\end{array}$ \\
\hline
\end{tabular}

Table 2: Balloon sizing at each simulated inflation pressure

\begin{tabular}{|c|c|c|}
\hline $\begin{array}{c}\text { Inflation Pressure } \\
(\mathbf{a t m})\end{array}$ & $\begin{array}{c}\text { Corresponding Rated Diameter } \\
(\mathbf{m m})\end{array}$ & $\begin{array}{c}\text { Balloon Sizing } \\
\text { (Balloon/Artery ratio) }\end{array}$ \\
\hline 10 & 2.8 & 0.933 \\
\hline 12 & 3.02 & 1.007 \\
\hline 14 & 3.2 & 1.067 \\
\hline
\end{tabular}


Table 3: Material parameters of the lipid and calcified plaques and their corresponding arteries

\begin{tabular}{|c|c|c|c|c|}
\hline Plaque Type & $\begin{array}{c}\text { Young Modulus of } \\
\text { Elasticity E (MPa) }\end{array}$ & $\begin{array}{c}\text { Poisson Ratio } \\
(\mathbf{v})\end{array}$ & $\begin{array}{c}\text { Yield Strength } \\
\text { (KPa) }\end{array}$ & $\begin{array}{c}\text { Tangent } \\
\text { Modulus (KPa) }\end{array}$ \\
\hline Lipid Plaque & 0.105 & 0.40 & 5 & 62.77 \\
\hline Calcified Plaque & 0.189 & 0.40 & 15.79 & 111.88 \\
\hline $\begin{array}{c}\text { Artery } \\
\text { (Lipid plaque case) }\end{array}$ & 0.677 & 0.44 & 7 & 256.5 \\
\hline $\begin{array}{c}\text { Artery } \\
\text { (Calcified plaque case) }\end{array}$ & 0.677 & 0.44 & 7 & 330.75 \\
\hline
\end{tabular}

Table 4: Relative \% error between experimental and FE simulations diameters obtained at respective pressures during inflation of the semi-compliant balloon described in section a. Green and blue cells also indicate diameters at nominal and rated burst pressures $\left(P_{N}\right.$ and $\left.P_{R B}\right)$ respectively

\begin{tabular}{|c|c|c|c|}
\hline $\begin{array}{c}\text { Pressure } \\
{\left[\boldsymbol{P}_{\boldsymbol{n}}\right]} \\
\mathbf{( a t m})\end{array}$ & $\begin{array}{c}\text { Diameter }\left[\boldsymbol{D}_{\boldsymbol{n}}\right] \\
\mathbf{( m m )}\end{array}$ & $\begin{array}{c}\text { Simulated } \\
\text { Diameter } \\
(\mathbf{m m})\end{array}$ & $\begin{array}{c}\text { \% Error on } \\
\text { Diameter }\end{array}$ \\
\hline $\mathbf{8}$ & 2.60 & 2.569 & 1.18 \\
\hline $\mathbf{9}$ & 2.65 & 2.691 & 1.55 \\
\hline $\mathbf{1 0}$ & 2.80 & 2.805 & 0.17 \\
\hline $\mathbf{1 1}$ & 2.91 & 2.912 & 0.07 \\
\hline $\mathbf{1 2}$ & 3.02 & 3.013 & 0.22 \\
\hline $\mathbf{1 3}$ & 3.10 & 3.110 & 0.32 \\
\hline $\mathbf{1 4}$ & 3.20 & 3.202 & 0.06 \\
\hline $\mathbf{1 5}$ & 3.27 & 3.290 & 0.61 \\
\hline $\mathbf{1 6}$ & 3.32 & 3.374 & 1.64 \\
\hline $\mathbf{1 7}$ & 3.43 & 3.456 & 0.75 \\
\hline $\mathbf{1 8}$ & 3.53 & 3.534 & 0.12 \\
\hline $\mathbf{1 9}$ & 3.60 & 3.610 & 0.28 \\
\hline $\mathbf{2 0}$ & 3.70 & 3.684 & 0.44 \\
\hline
\end{tabular}




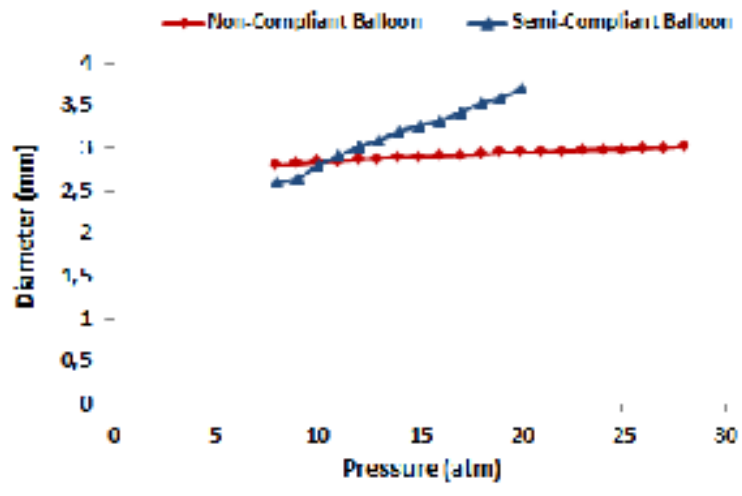




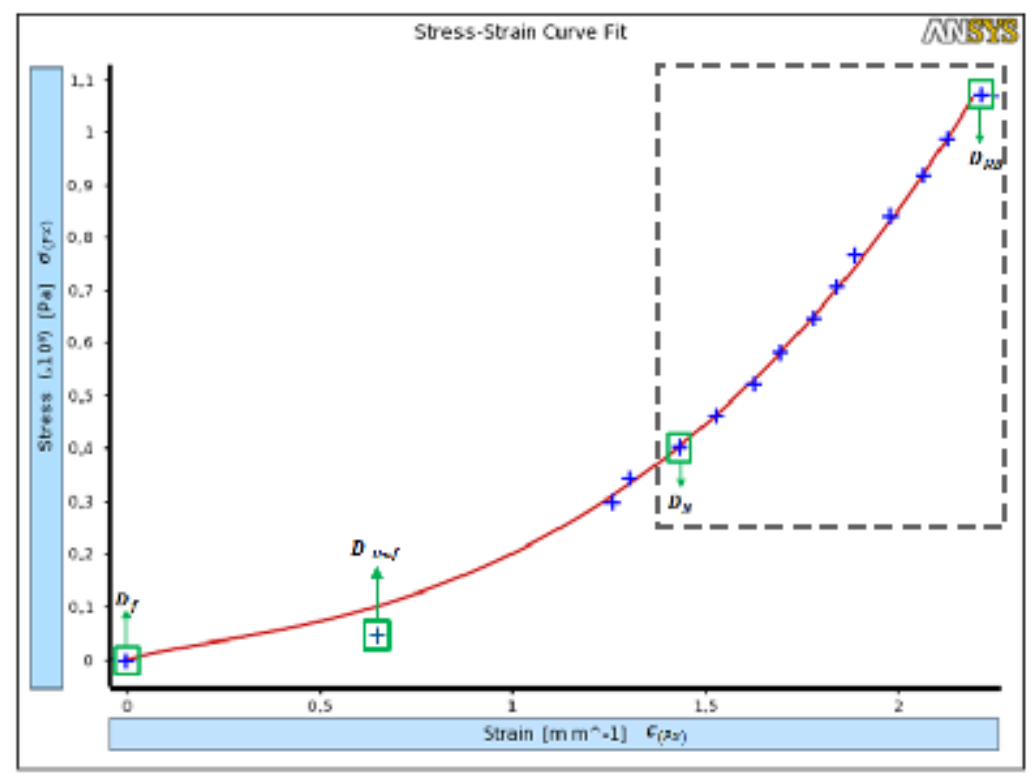




\section{Balloon Catheter Geometry}

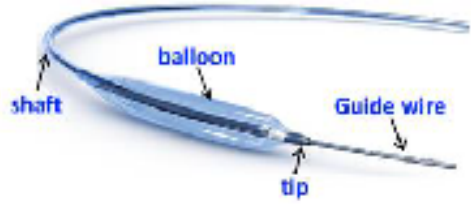




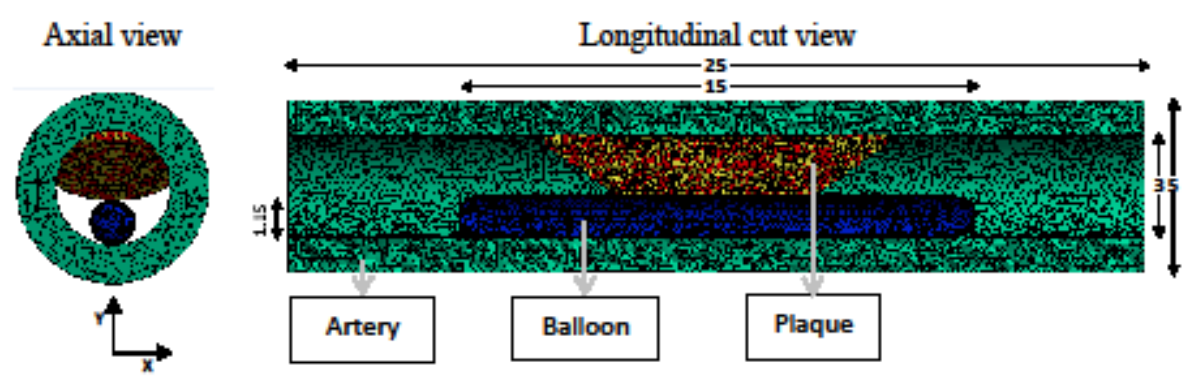




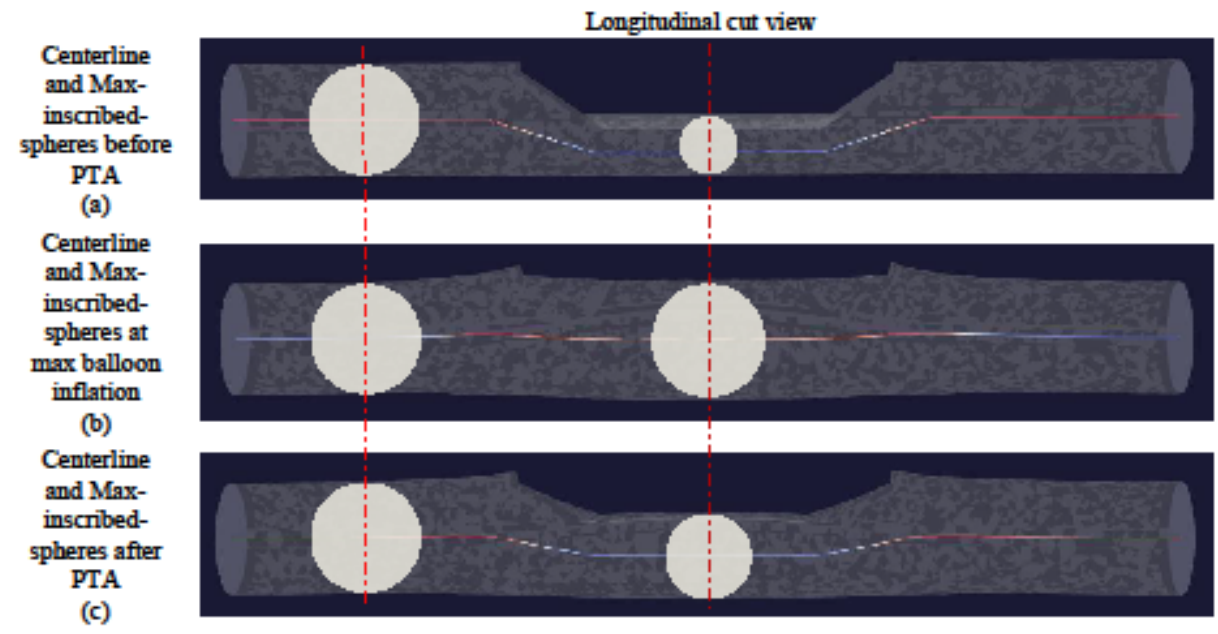


(A-I): Axial view

(A-II): Plastic deformations in stenosed artery (longitudinal cut view)
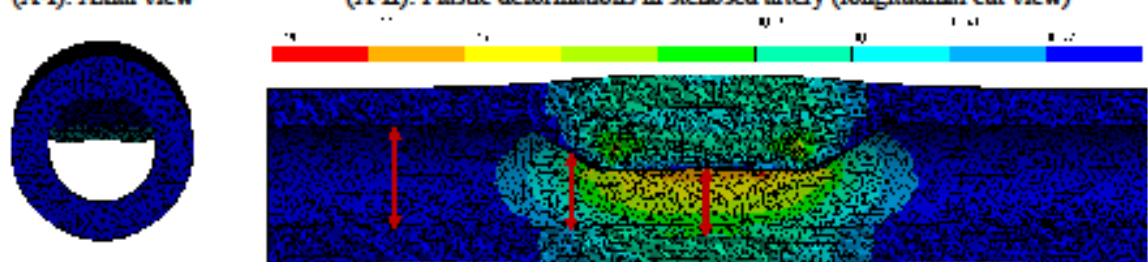

(B-I): Semi-compliant balloon profile at max inflation

(B-II): Non-compliant balloon profile at max inflation
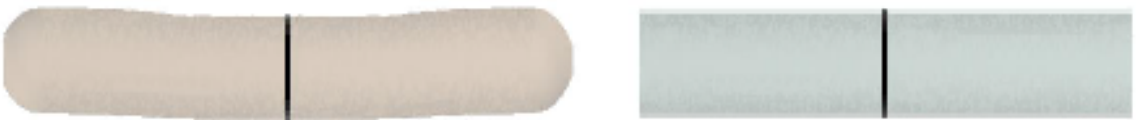

(C): Semi vs. Non-conpliant balloons diametric evolution at their central planes simulated outside and inside stenosed arteries

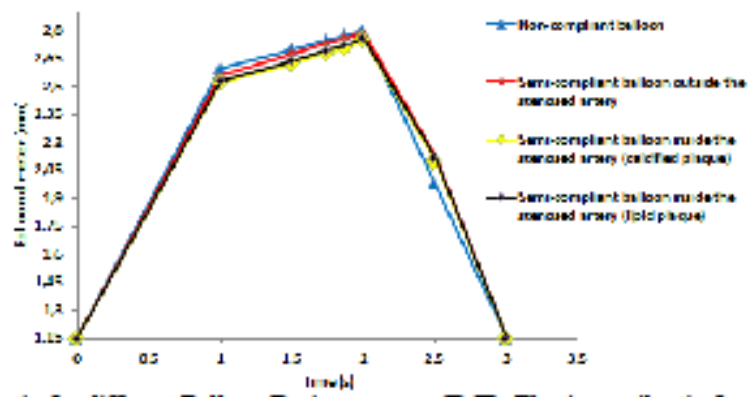

(D-I): Lumen gain ratio for different Balloon Designs, $\quad$ (D-II): Elastic recoil ratio for different Balloon and the initial humen diameter distribution

$$
\text { Designs }
$$
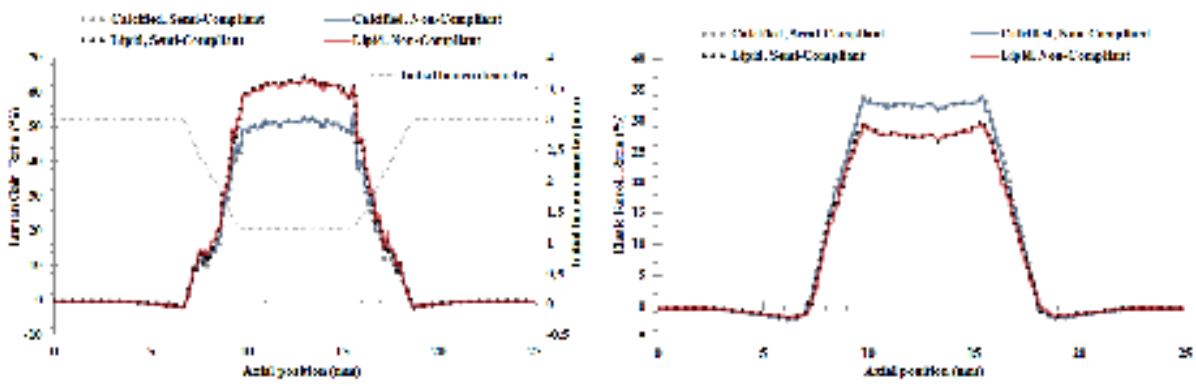


\section{Lumen gain ratio for different Plaque Materia Compositions, and the initial lumen diameter distribution}

(A)

Elastic recoil ratio for different Plaque Material Compositions

(B)
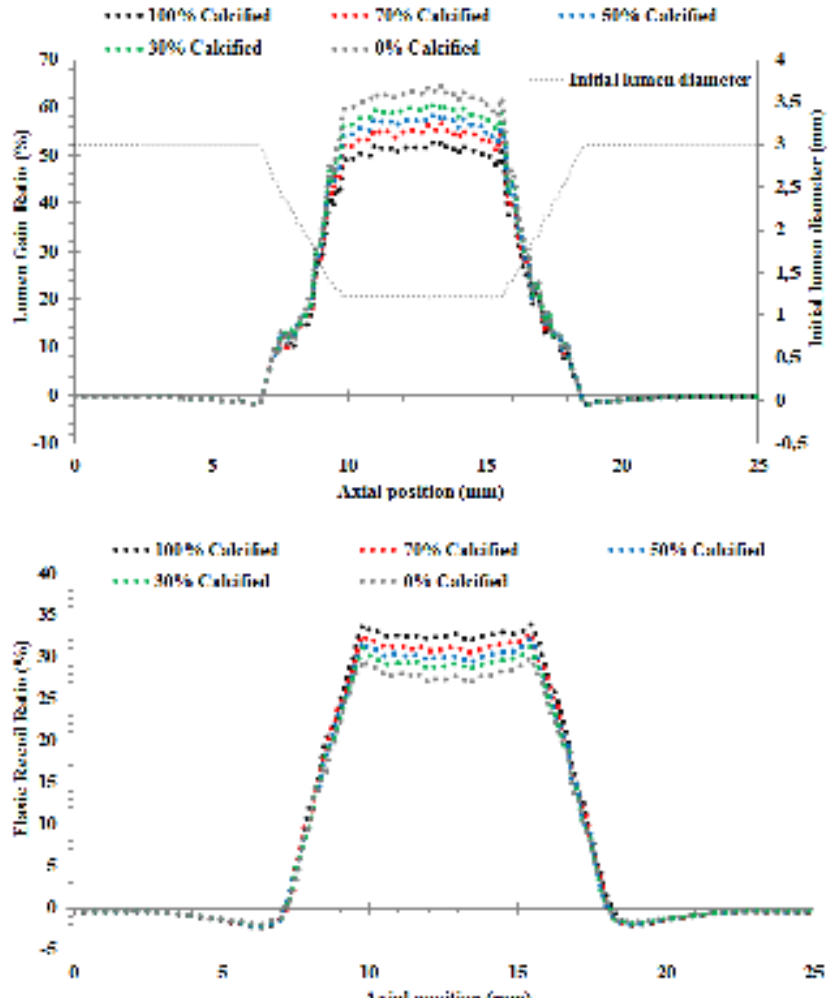
Lumen gain ratio for different Balloon Sizings,

and the initial lumen

diameter distribution

(A)

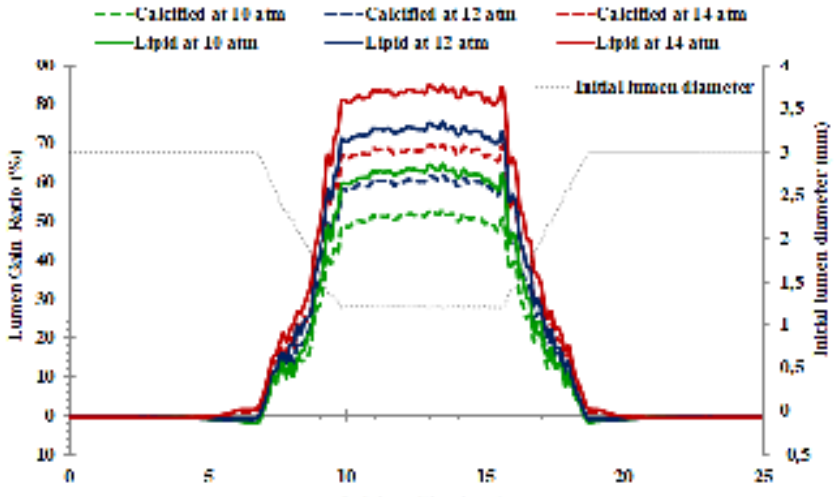

Jxtal posteton (mm)

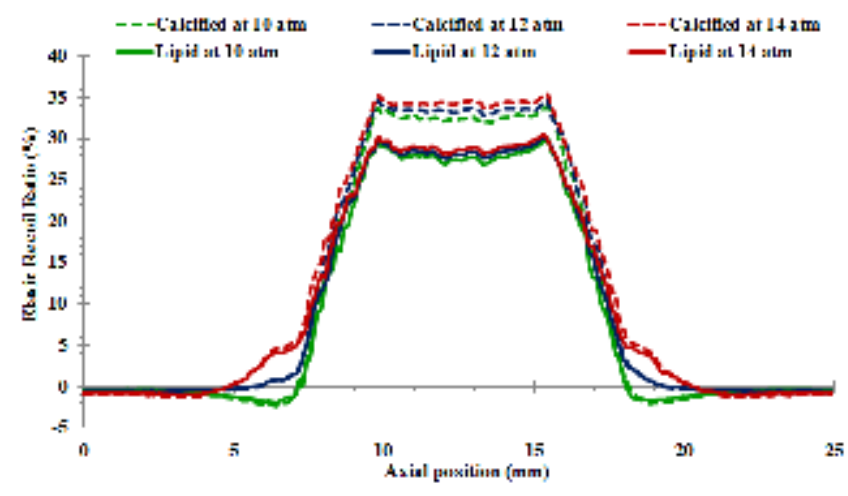

Elastic recoil ratio for different Balloon Sizings

(B)

Balling at

$10 \mathrm{~atm}$

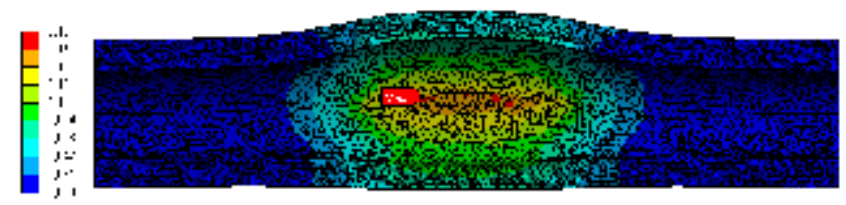

(C)

Balloon Sizing at
$12 \mathrm{~atm}$

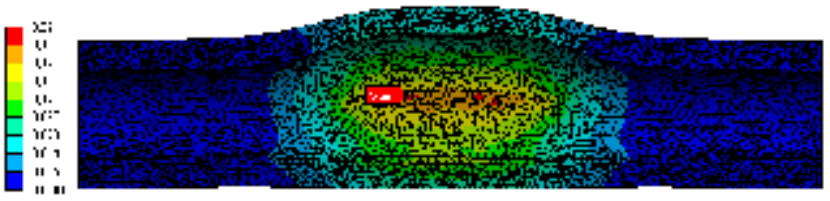

Balloon Sizing at

$14 \mathrm{~atm}$
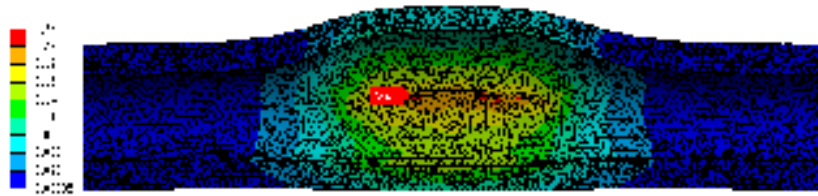


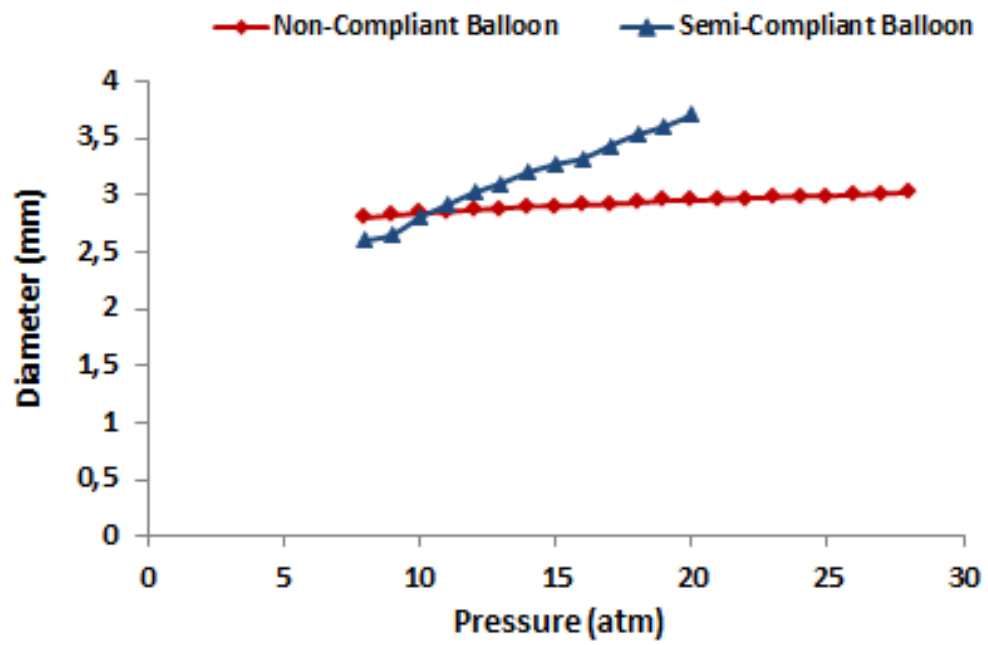




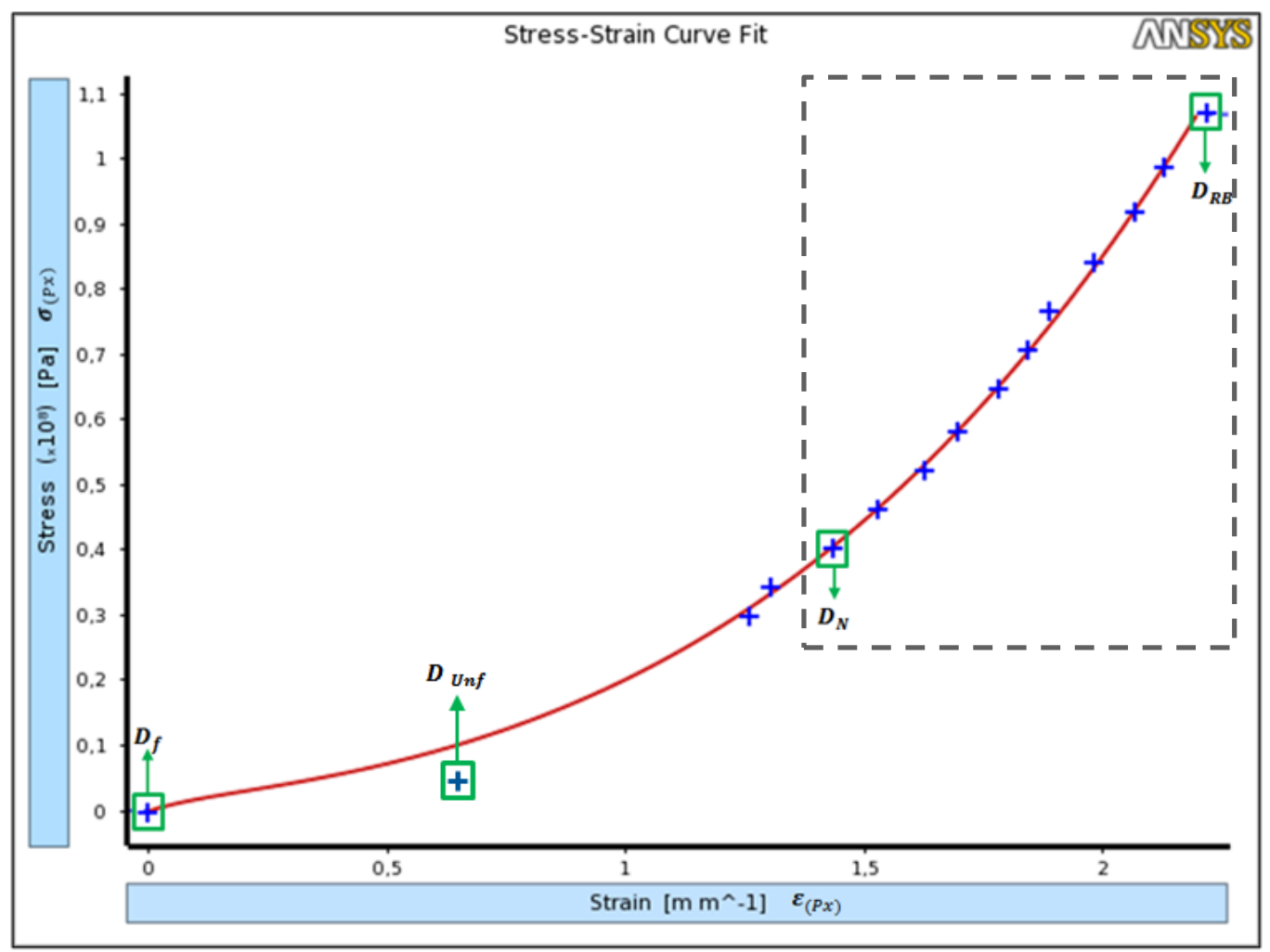




\section{Balloon Catheter Geometry}

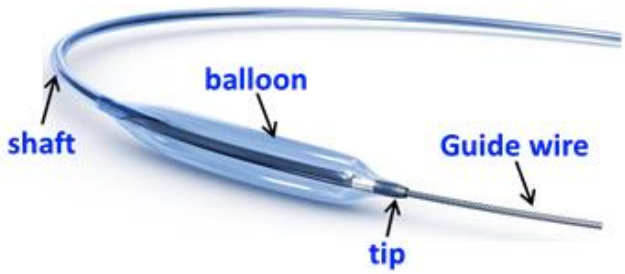




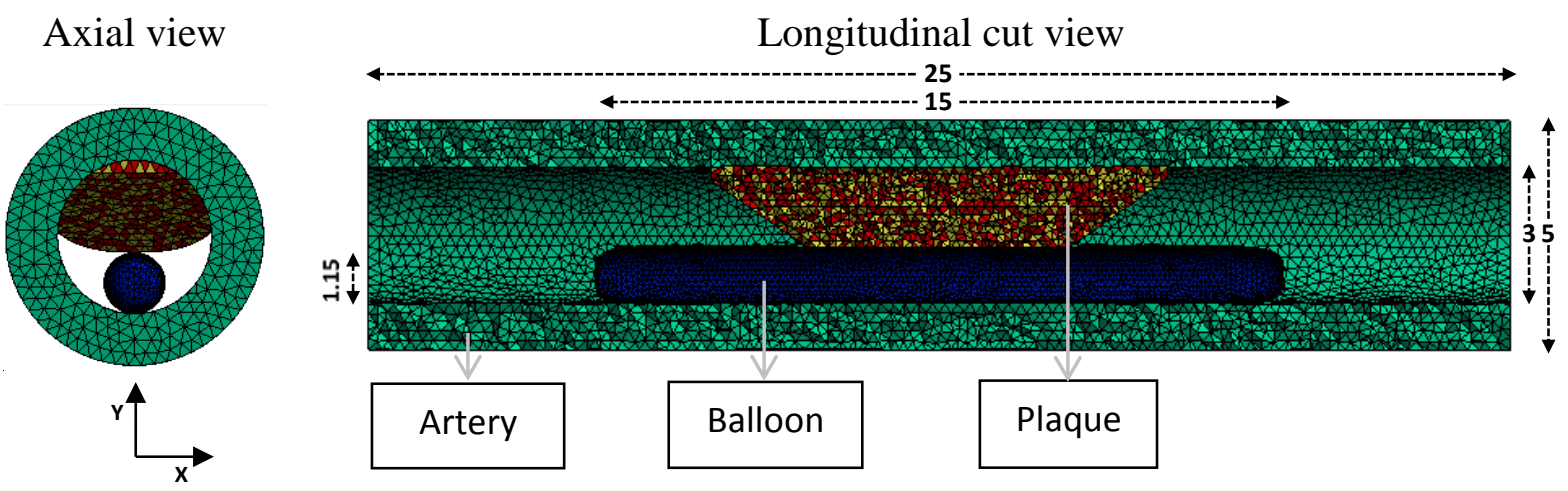




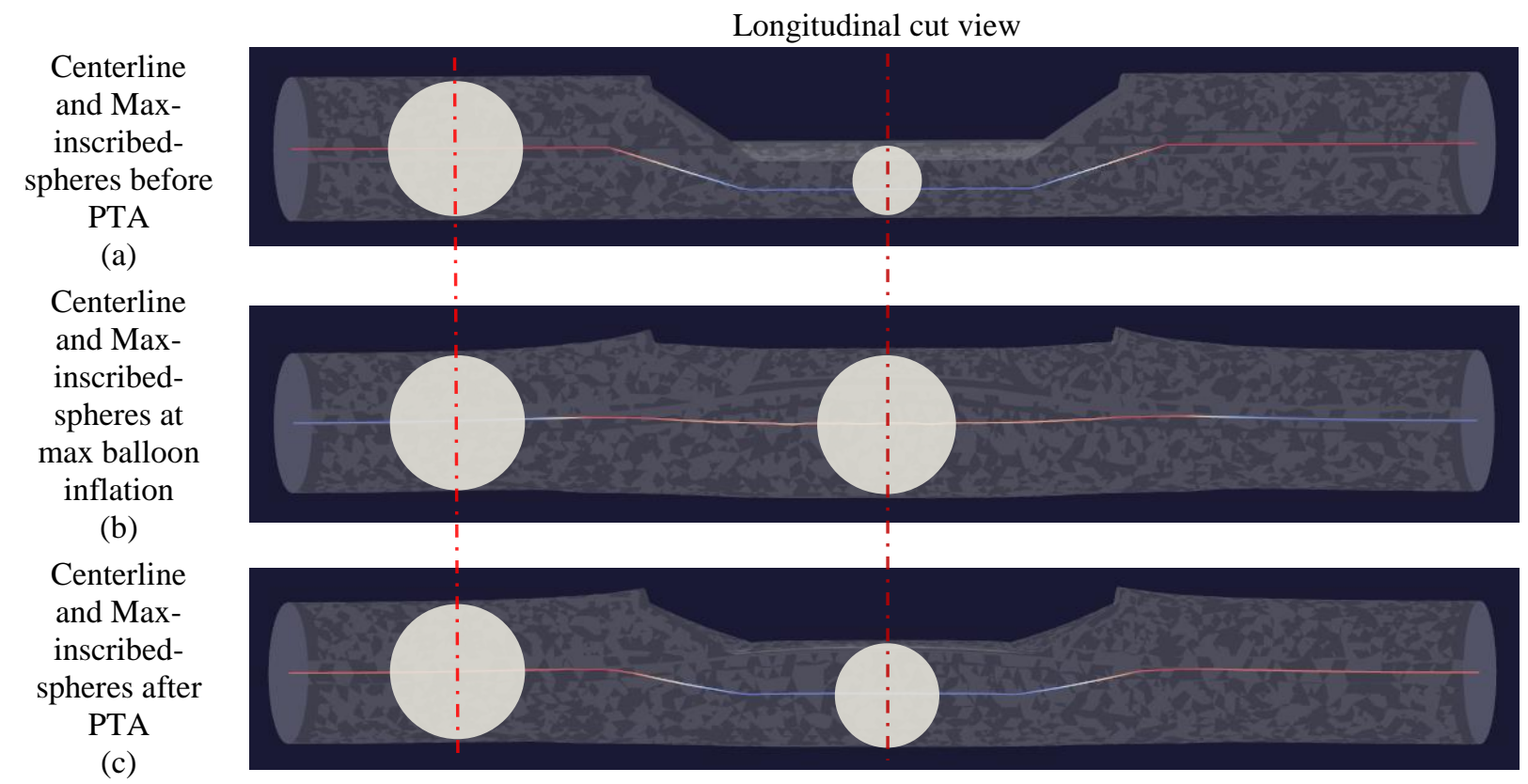


(A-I): Axial view

(A-II): Plastic deformations in stenosed artery (longitudinal cut view)
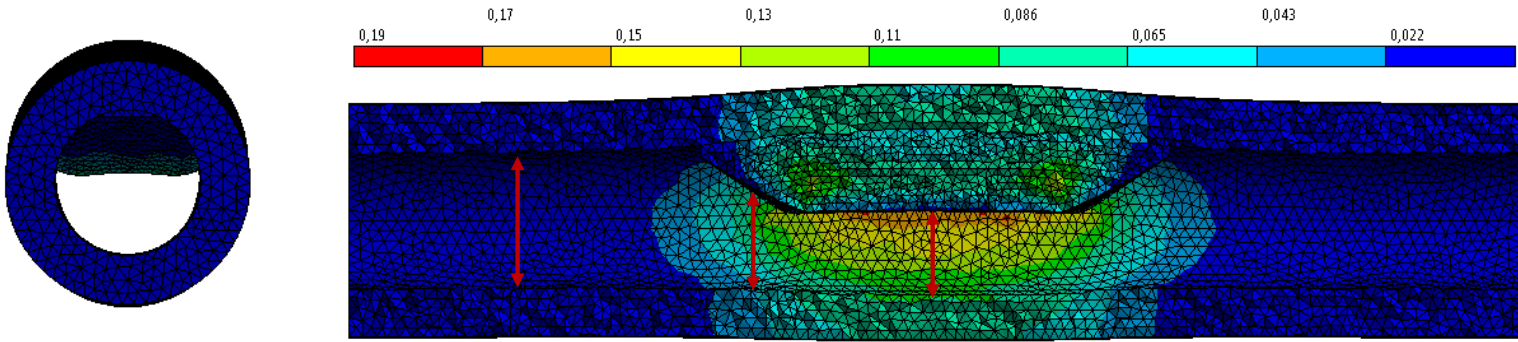

(B-I): Semi-compliant balloon profile at max inflation

(B-II): Non-compliant balloon profile at max inflation
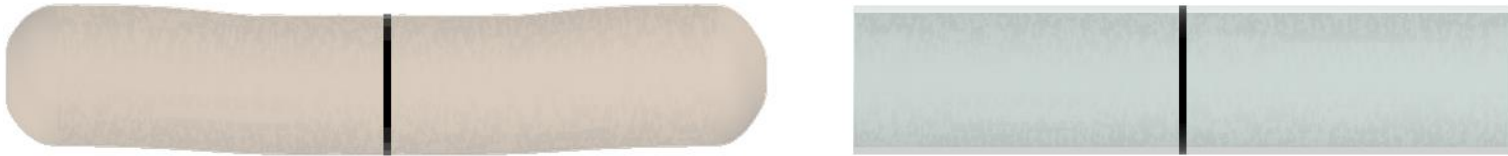

(C): Semi vs. Non-compliant balloons diametric evolution at their central planes simulated outside and inside stenosed arteries

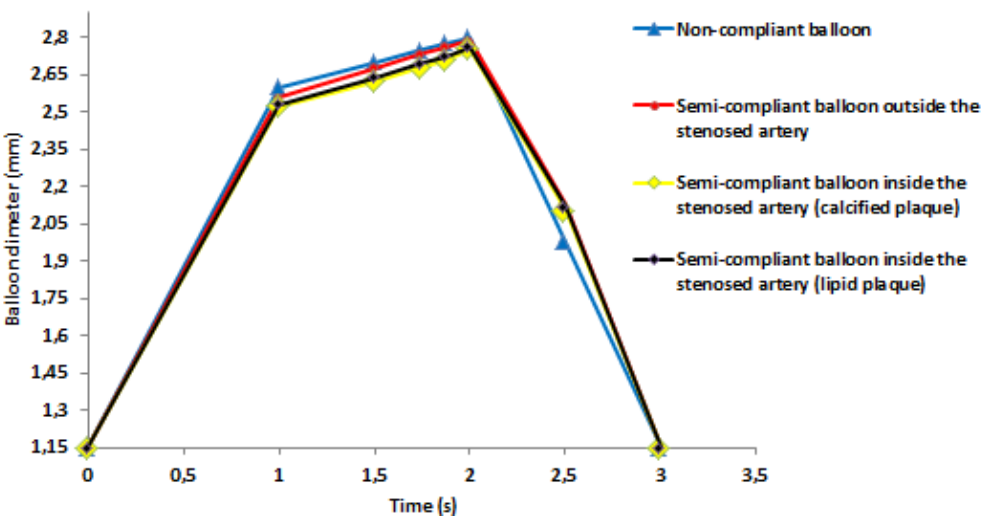

(D-I): Lumen gain ratio for different Balloon Designs, and the initial lumen diameter distribution

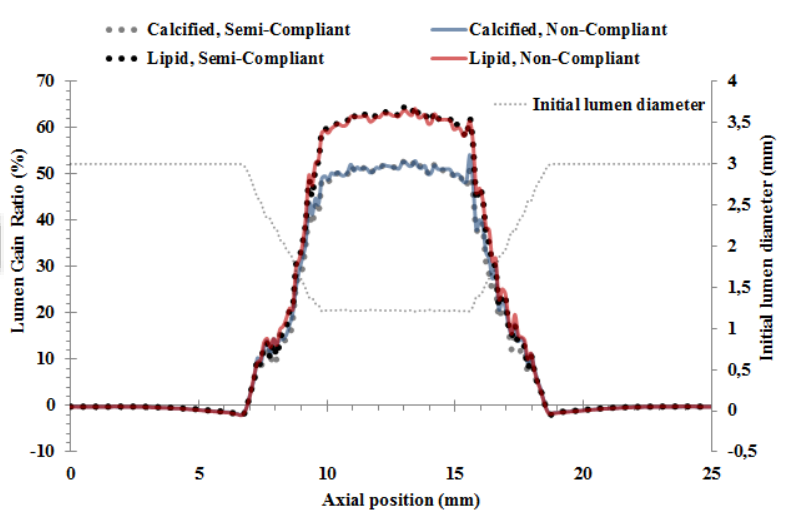

Designs

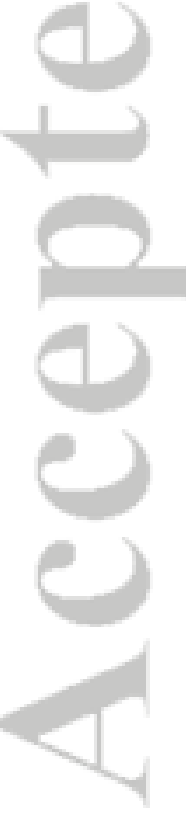

(D-II): Elastic recoil ratio for different Balloon

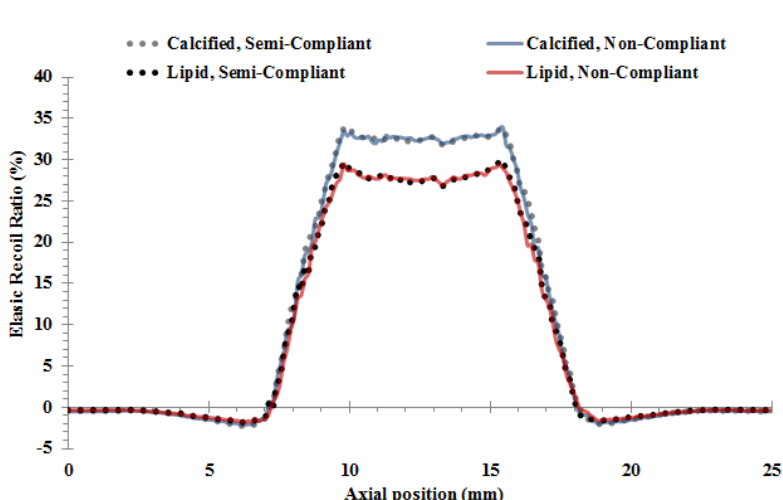


Lumen gain ratio for different Plaque Material Compositions, and the initial lumen diameter distribution

(A)

Elastic recoil ratio for different Plaque Material Compositions (B)
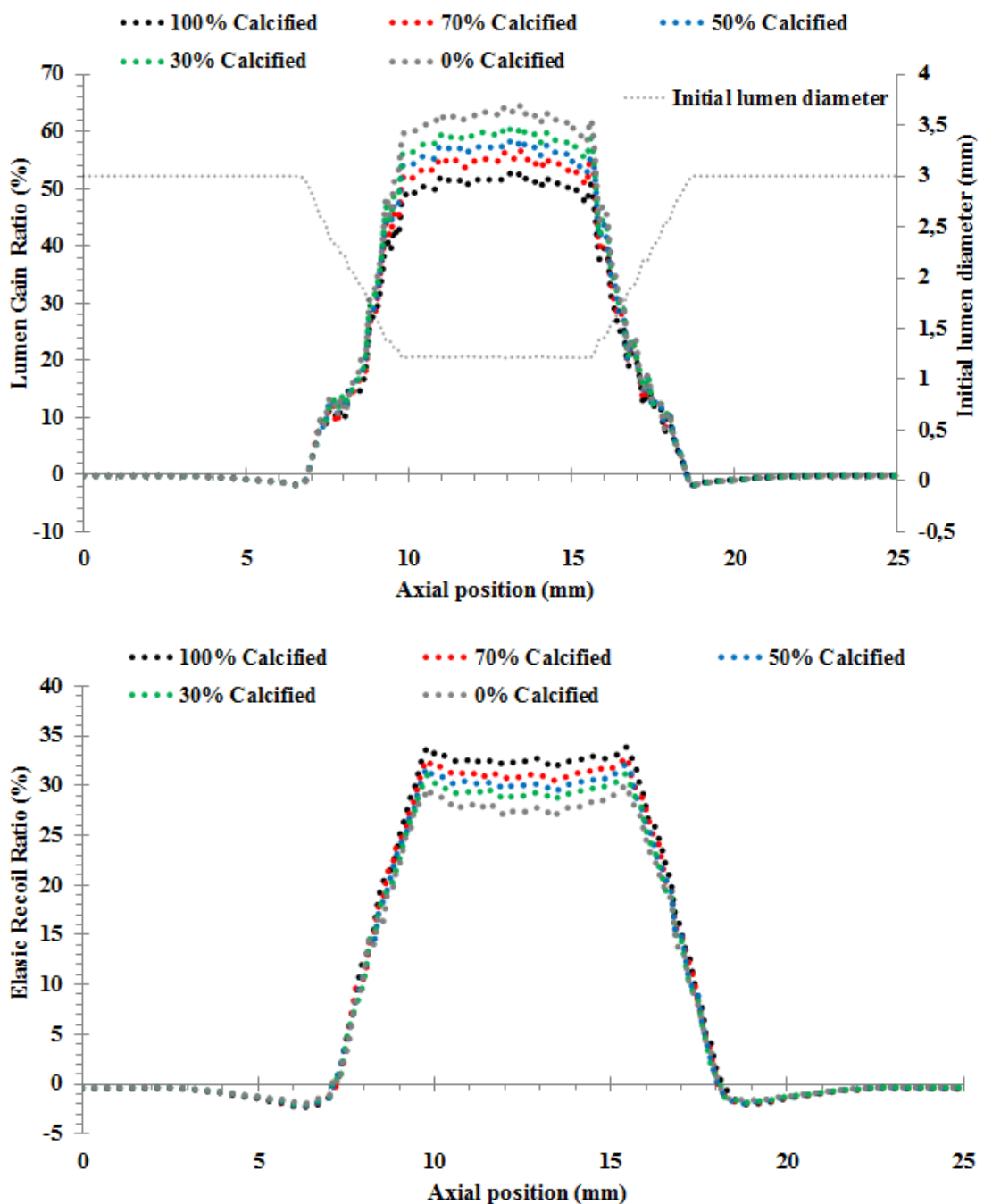
Lumen gain ratio for different Balloon Sizings, and the initial lumen diameter distribution (A)

Elastic recoil ratio for different Balloon Sizings

(B)

Balloon Sizing at $10 \mathrm{~atm}$

(C)

Balloon Sizing at $12 \mathrm{~atm}$

Balloon Sizing at $14 \mathrm{~atm}$
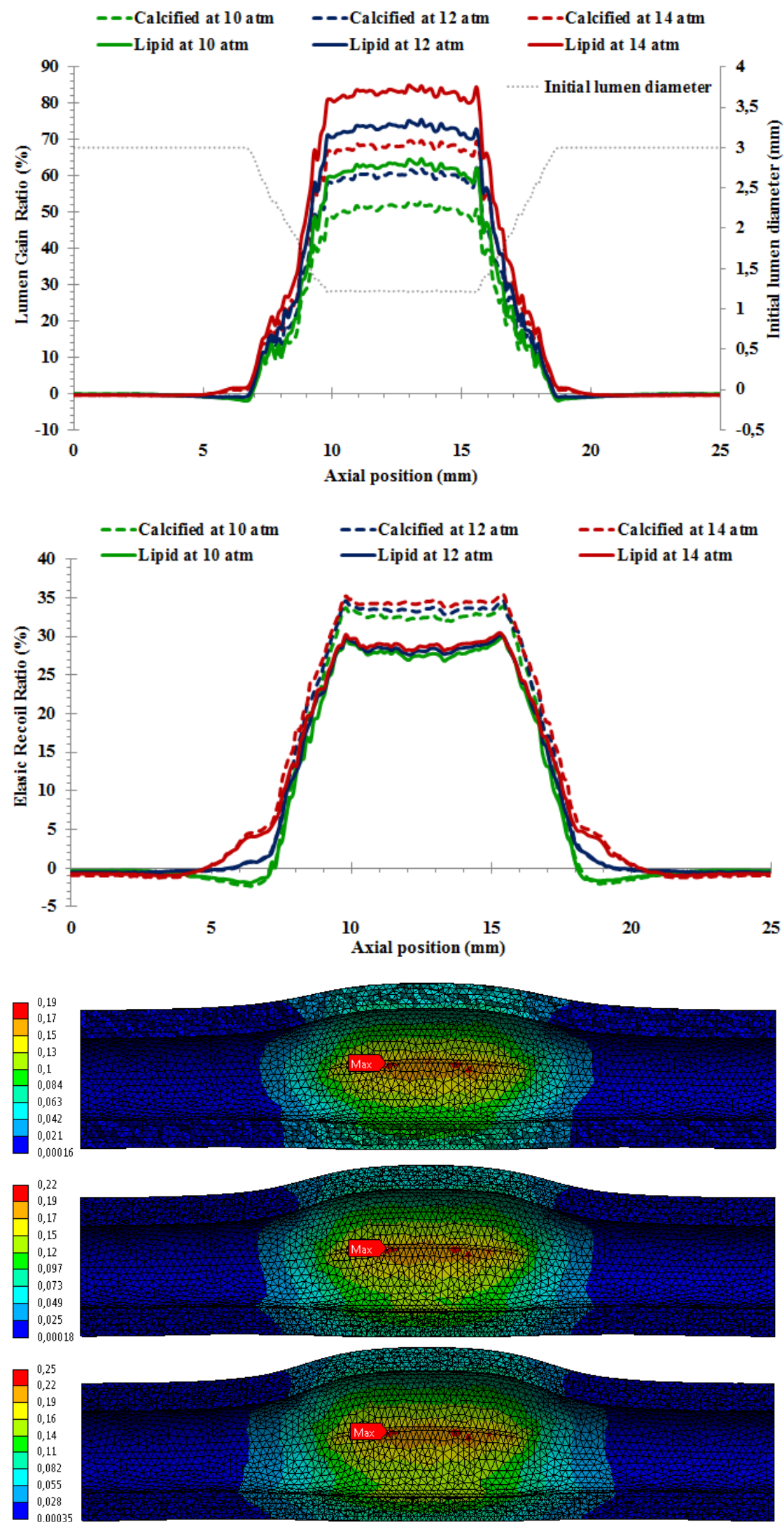
Lipid plaque

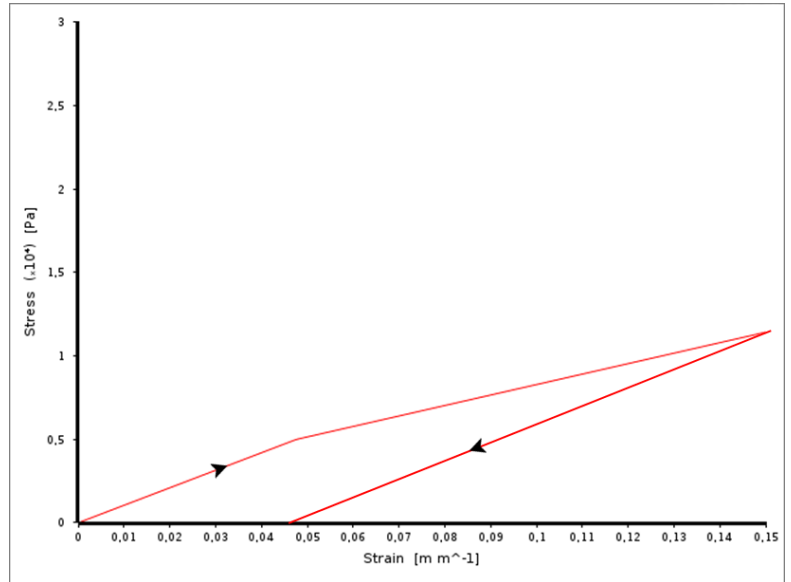

Calcified plaque

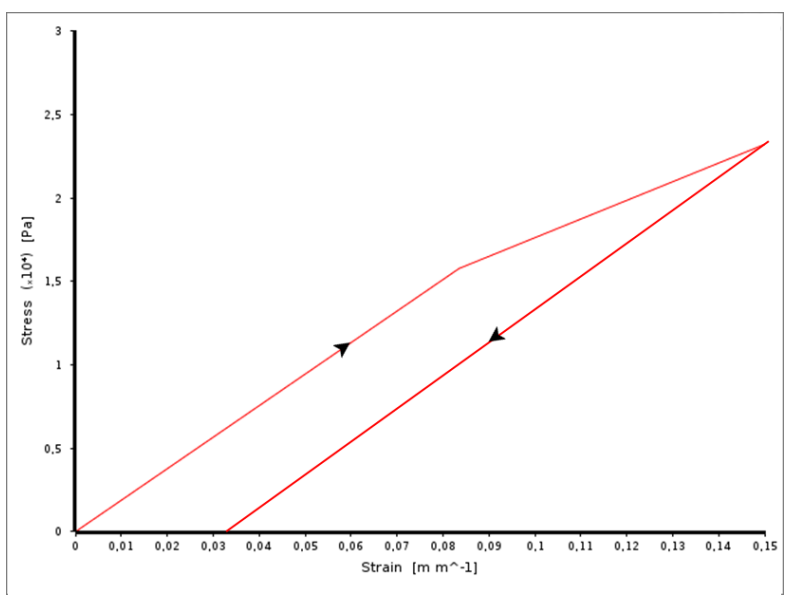


Artery

(Lipid plaque case)

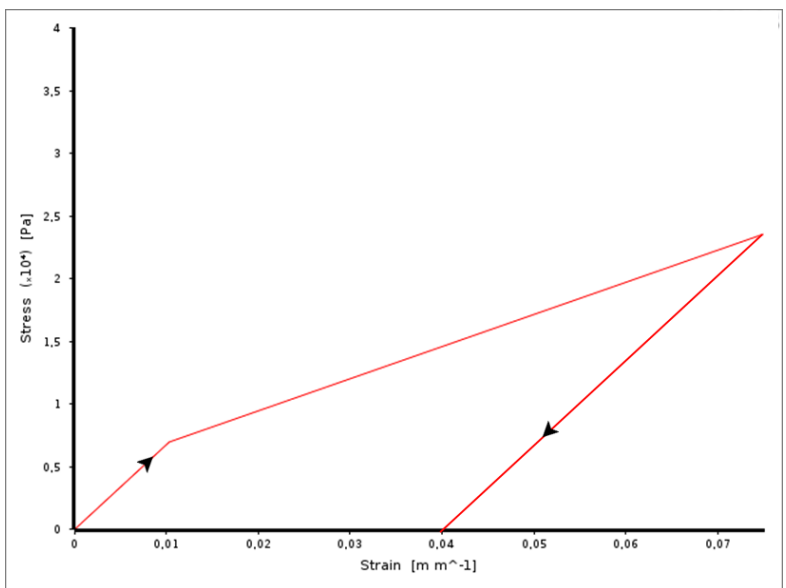

Artery

(Calcified plaque case)

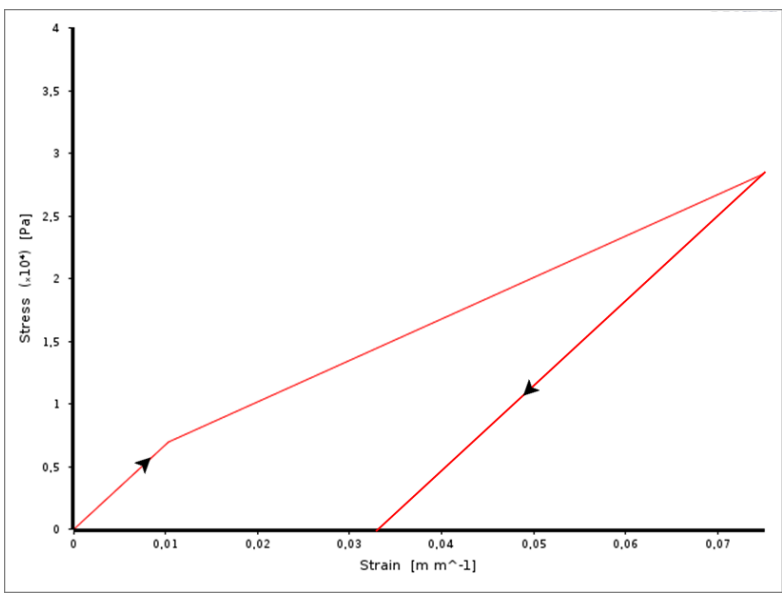


A
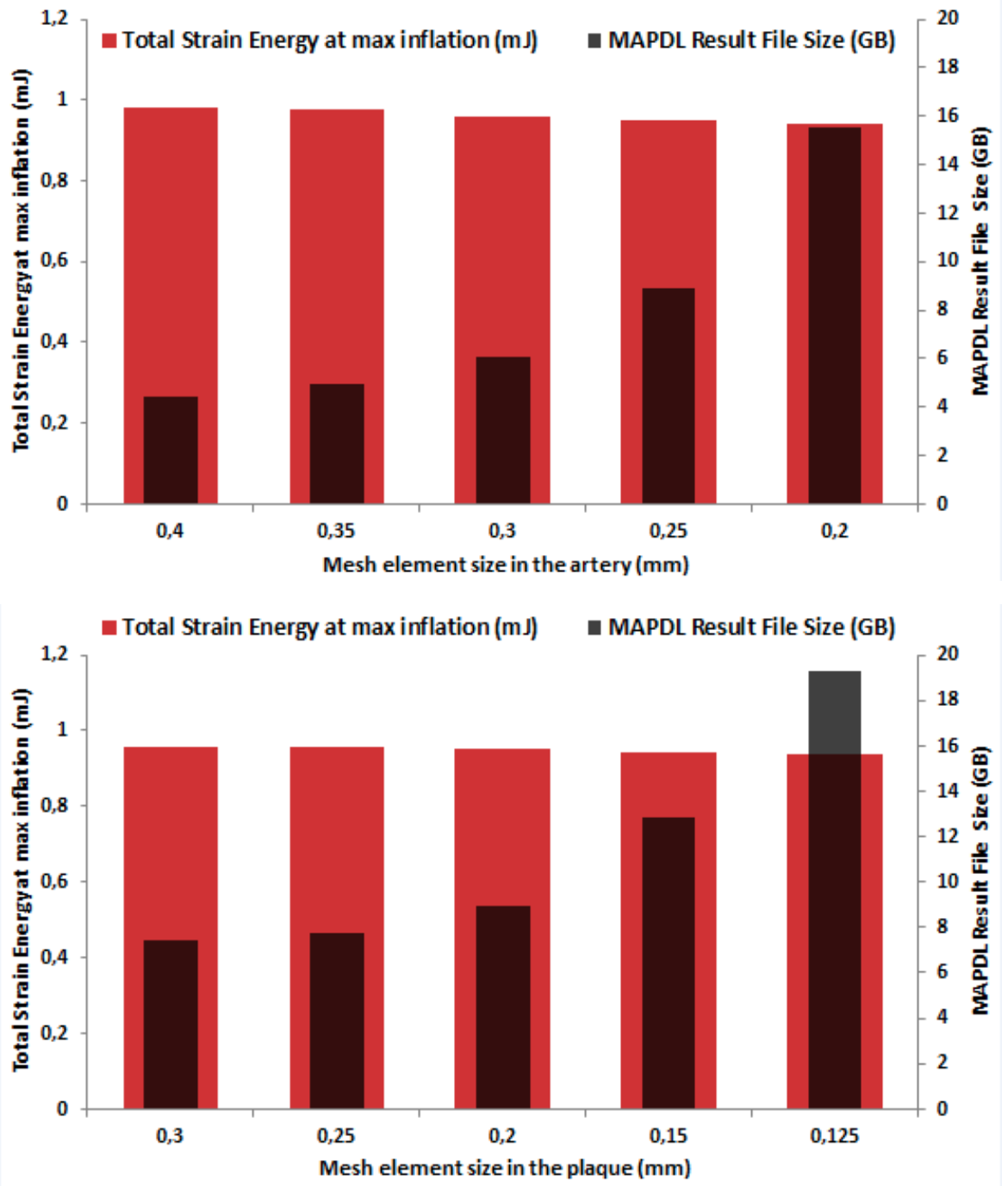
Artery: $0.4 \mathrm{~mm}$

Plaque: $0.2 \mathrm{~mm}$

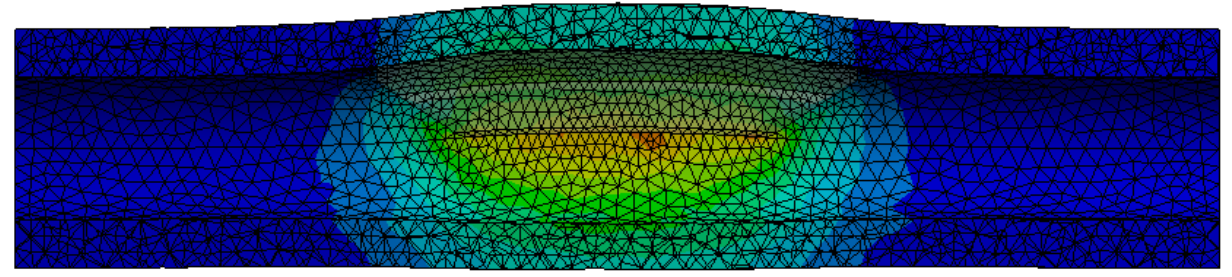

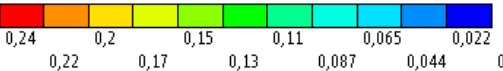

Artery: $0.35 \mathrm{~mm}$

Plaque: $0.2 \mathrm{~mm}$

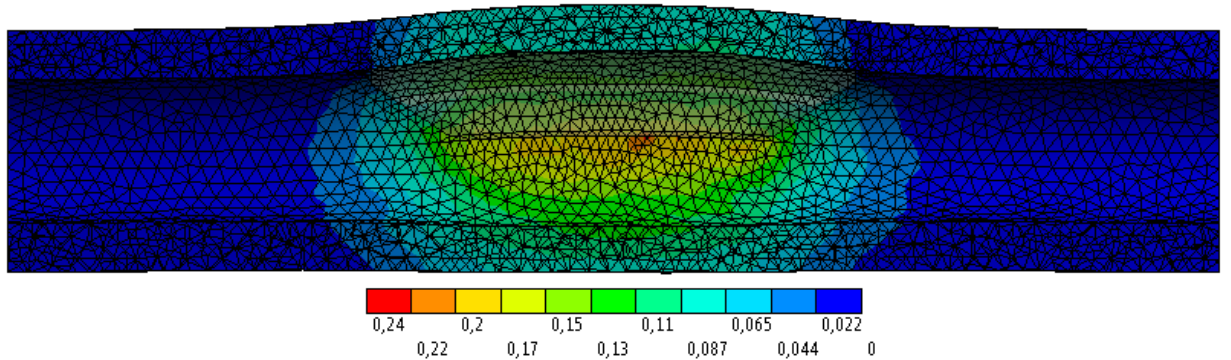

Artery: $0.25 \mathrm{~mm}$

Plaque: $0.3 \mathrm{~mm}$
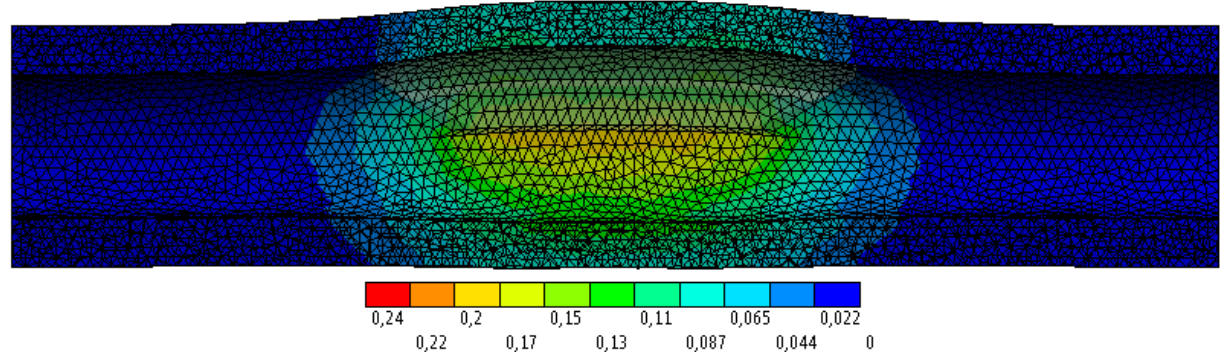

Artery: $0.25 \mathrm{~mm}$

Plaque: $0.2 \mathrm{~mm}$
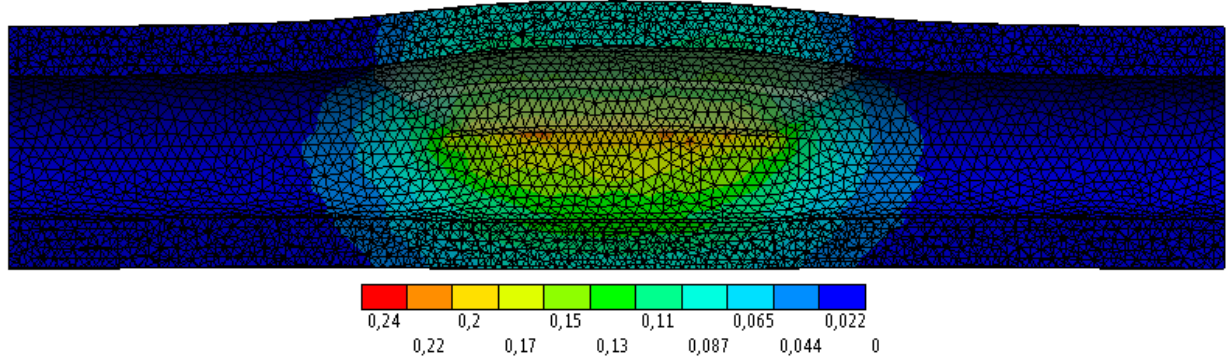

Artery: $0.25 \mathrm{~mm}$

Plaque: $0.15 \mathrm{~mm}$
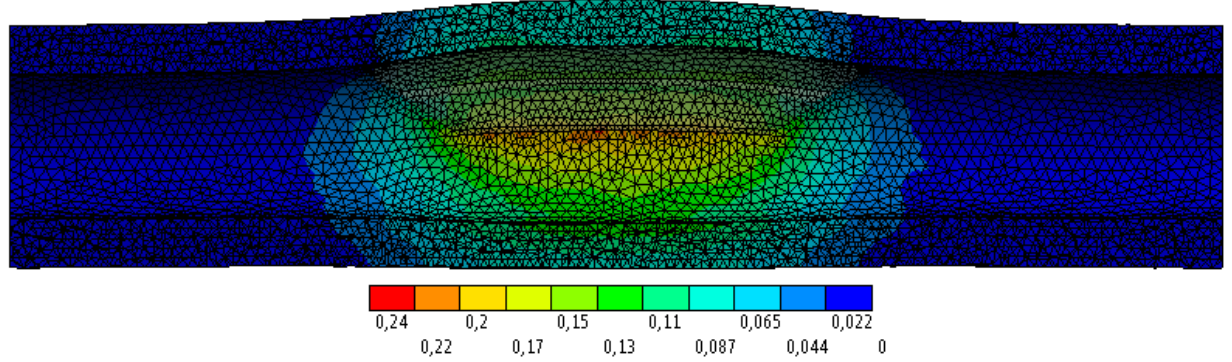

Artery: $0.2 \mathrm{~mm}$

Plaque: $0.2 \mathrm{~mm}$
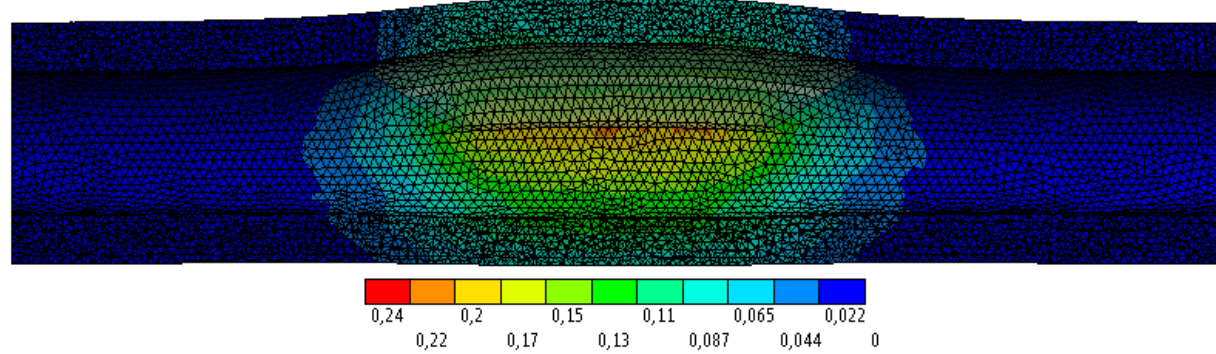